\title{
Computable genuine multimode entanglement measure: Gaussian vs. non-Gaussian
}

\author{
Saptarshi Roy ${ }^{1}$, Tamoghna Das ${ }^{2}$, Aditi Sen $(D e)^{1}$ \\ ${ }^{1}$ Harish-Chandra Research Institute, HBNI, Chhatnag Road, Jhunsi, Allahabad 211 019, India and \\ ${ }^{2}$ International Centre for Theory of Quantum Technologies, University of Gdańsk, 80-952 Gdańsk, Poland.
}

\begin{abstract}
Genuine multimode entanglement in continuous variable systems can be quantified by exploring the geometry of the state-space, namely via the generalized geometric measure (GGM). It is defined by the shortest distance of a given multimode state from a nongenunely multimode entangled state. For the multimode Gaussian states, we derive a closed form expression of GGM in terms of the symplectic eigenvalues of the reduced states. Following that prescription, the characteristics of GGM for typical three- and four-mode Gaussian states are investigated. In the non-Gaussian paradigm, we compute GGM for photon-added as well as -subtracted states having three- and four-modes and find that both addition and subtraction of photons enhance the genuine multimode entanglement of the state compared to its Gaussian counterpart. Our analysis reveals that when an initial three-mode vacuum state is evolved according to an interacting Hamiltonian, photon addition is more beneficial in increasing GGM compared to photon subtraction while the scenerio reverses when one considers the four mode non-Gaussian states. Specifically, subtracting photons from four-mode squeezed vacuum states almost always result in higher multimode entanglement content than that of photon addition to both single as well as multimode and constrained as well as unconstrained operations. Furthermore, we observe that GGM freezes under subtraction of photons involving multiple modes, in some specific cases. This feature is novel in its own rights as it does not appear while adding photons. Finally, we relate the enhancements of GGM with the distance-based non-Gaussianity measure.
\end{abstract}

\section{INTRODUCTION}

Among the plethora of nonclassical features, intrinsic to quantum mechanics, entanglement [1], a term coined by Schrodinger [2], is arguably the most fascinating one. Entanglement, captures the degree of inseparability of quantum systems, turns out to be the resource exhibiting "quantum advantage and supremacy" in various quantum information processing tasks like teleportation [3-5], dense coding $[6,7]$, entanglement-based quantum cryptography $[8,9]$ and the detection of quantum phase transitions [10-13], to name a few.

Classification of quantum systems based on entanglement is one of the premiere endeavors in quantum information science. Although the problem of quantifying entanglement of a given system (state) turns out to be more intricate with the increase of the number of parties. However, if one restricts the analysis only for pure states, the categorization becomes somehow simpler. For example, bipartite pure states can either be entangled or product. For multiple parties, even within the set of pure states, we can have diverse possibilities, where states can be entangled for some of the parties while product with the rest. A prototypical instance of such a situation can be illustrated with an example of a three-qubit state, $|\eta\rangle_{A B C}=\left|\psi^{-}\right\rangle_{A B} \otimes|\phi\rangle_{C}$, commonly known as a biseparable state, where $\left|\psi^{-}\right\rangle_{A B}$ is the maximally entangled state and $|\phi\rangle_{C}$ is any arbitrary pure qubit. Note that $|\eta\rangle_{A B C}$ is product in $A B: C$, and the reduced $A: C$ as well as $B$ : $C$ bipartitions, while it is entangled in other bipartite cuts. The pure quantum states that are entangled in all bipartitions are called genuinely multiparty entangled. Typically, different kinds of entanglement present in multiparty systems can be broadly quantified in two ways: using distance-based (geometric) measures [14-24], and by using monogamy-based measures [25-36]. In finite dimensions, genuine multipartite entanglement (GME) for pure states can be computed efficiently by a distancebased measure called the generalized geometric measure (GGM) [37] (see also [38-41]), and has been used extensively to study genuine multipartite entanglement for a wide range of quantum systems [42-46]. Although attempts have been made to generalize GGM for mixed states [47], the analysis is not exhaustive and only works for some specific classes of states.

In this paper, we are interested in investigating the entanglement between various modes of multimode states of light, where each mode contains an arbitrary number of photons. Hence, the corresponding Hilbert space is an infinite dimensional one [48-50]. We first show that, like in finite dimensions, the GGM can still be simplified in terms of the Schmidt coefficients. However, this route is not very efficient for continuous variable systems since the operations involve infinite dimensional matrices. For Gaussian states [51, 52], we show that such problems can be resolved by expressing it in a closed analytical form in terms of the symplectic invariants, namely the symplectic eigenvalues of the covariance matrix. These invariants can be easily computed from the quadratic quadrature correlations, which form the elements of the covariance matrix. Since the dimension of the covariance matrix grows linearly with the number of modes, our method provides an efficient and scalable prescription for computing GGM of pure Gaussian states having an arbitrary number of modes.

We demonstrate the applicability of our recipe by computing the GGM for some prototypical Gaussian states of three- and four-modes. Examples include three- and 
four-mode squeezed states in which GGM increases with the increase of the squeezing strength. We also show an instance of generating genuine multimode entanglement from an initially uncorrelated three-mode vacuum state which is evolved by an interacting Hamiltonian describing a nonlinear crystal. We want to stress here that our prescription is lucrative from an experimental point of view since the symplectic eigenvalues can be computed from the data of the quadrature correlations composing the covariance matrix.

We then move on to study genuine multimode entanglement in non-Gaussian states. Although the Gaussian states offer several advantages like elegant mathematical simplicity in the description and easy experimental realizability. It has also been established that non-Gaussian resources are "more" useful compared to their Gaussian counterparts for some of the quantum information protocols including bosonic codes [53], pioneering application in photonic quantum computation [54], quantum metrology [55], entanglement distillation [56, 57], entanglement distribution [58], error correction [59], phase estimation [60], quantum communication [61] and quantum cloning [62].

In general, any state whose Wigner function [63] is not Gaussian in the quantum phase space is considered as a non-Gaussian state. One of the popular methods to obtain non-Gaussian states is to add or subtract photons in different modes of a Gaussian state. It has also been demonstrated that photon addition and subtraction make a negative dip [64] in the Gaussian Wigner function of a Gaussian state, while the entanglement of the photon-added and -subtracted states are always monotonically increasing with the number of photon added and subtracted [65, 66].

In this paper, we also study whether de-Gaussification leads to the enhancement of GGM from its Gaussian value. Among the different de-Gaussification techniques, we choose the method of photon addition and subtraction since it can be scalably applied when the the number of modes of the initial Gaussian state grows and can also be achieved experimentally [67, 68]. Specifically, we add or subtract photons from different modes of the three- and four-mode Gaussian states and track the enhancement of GGM. In our context of computation of genuine multimode entanglement, we find that both adddition and subtraction of photons in one or several modes of the squeezed vacuum state lead to enhancement in the GGM content compared to its Gaussian counterpart. Further analysis reveal that photon-subtraction leads to a higher multimode entanglement than that of the photon-added state. Moreover, we choose the relative entropy-based non-Gaussianity measure to quantify the departure of a given state from the initial Gaussian states and show that it increases monotonically with the increase of number of photons added (subtracted). We, however, notice that such a non-Gaussianity measure fails to shed light on the increment obtained for genuine multimode entanglement content of photon-subtracted state over the photon- added ones.

This work is organized as follows. After giving the definition of GGM in continuous variable systems in Sec. II, we show that the computation of GGM can be simplified both for Gaussian and non-Gaussian states. We then evaluate the expression of GGM for Gaussian states in terms of its symplectic invariants in Sec. III and obtain GGM for some typical examples in Sec. III A. The results involving non-Gaussian states are given in Sec. IV before presenting the conclusion in Sec. V.

\section{PRELIMINARIES}

We introduce the notion of genuine multimode entanglement measure based on the geometry of multimode quantum states. The generalized geometric measure (GGM) for an arbitrary $N$-mode pure quantum state, $\left|\psi_{12 \ldots N}\right\rangle$, is defined as

$$
\mathcal{G}\left(\left|\psi_{12 \ldots N}\right\rangle\right)=1-\max _{|\chi\rangle \in n G}\left|\left\langle\chi \mid \psi_{12 \ldots N}\right\rangle\right|^{2},
$$

where the maximization is taken over the set of all $N$ mode pure states $|\chi\rangle$, which are not genuinely multimode entangled, denoted by $n G$. The distance measure used in this case is the Fubini study metric [69, 70]. Based on Schmidt decomposition for continuous variable systems $[71,72]$ which can be easily extended for any normalizable infinite dimensional state (Apendix A for details), we obtain an expression of GGM in terms of the eigenvalues of the reduced density matrices. We call it to be the "canonical" form of GGM.

We then show in the next section that for Gaussian states, a much more elegant and efficient form of GGM in terms of symplectic eigenvalues of the reduced modes can be obtained. On the other hand, the canonical form of GGM can be important to quantify multimode entanglement for non-Gaussian states where the simplifications as obtained in the Gaussian case is unavailable. and we have to resort to the brute force method.

\section{A. The canonical formula of GGM}

We now use Schmidt decomposition to simplify the evaluation of GGM given in Eq. (1). Since $|\chi\rangle$ is nongenuinely multimode entangled, i.e., it is product with respect to at least one modal-bipartition, we can always write the state in the Schmidt decomposition across that partition as

$$
|\chi\rangle=\left|\chi_{12 \ldots N}\right\rangle=\left|\chi_{\mathcal{A}}\right\rangle \otimes\left|\chi_{\mathcal{B}}\right\rangle
$$

where we assume that $\mathcal{A}$ and $\mathcal{B}$ contains $n(\mathcal{A})$ and $n(\mathcal{B})$ modes respectively, such that $n(\mathcal{A})+n(\mathcal{B})=N$. By using the Schmidt decomposition in the same $\mathcal{A}: \mathcal{B}$ modal-bipartition, we can write the given state $\left|\psi_{12 \ldots N}\right\rangle$ 
of $n(\mathcal{A})+n(\mathcal{B})$ modes as

$$
\left|\psi_{12 \ldots N}\right\rangle=\sum_{i} \sqrt{\lambda_{i}}\left|\mu_{\mathcal{A}}^{i}\right\rangle \otimes\left|\nu_{\mathcal{B}}^{i}\right\rangle,
$$

where $\left\{\lambda_{i}\right\}$ are the set of Schmidt coefficients which are always positive and $\sum_{i} \lambda_{i}=1$. Here $\left\{\left|\mu_{A}^{i}\right\rangle\right\}$ and $\left\{\left|\nu_{\mathcal{B}}^{i}\right\rangle\right\}$ are the Schmidt basis (also form a basis ${ }^{1}$ in the Hilbert spaces of $\mathcal{A}$ and $\mathcal{B}$ ) and $i$ runs upto $\min \left\{\operatorname{dim} \mathcal{H}^{\mathcal{A}}, \operatorname{dim} \mathcal{H}^{\mathcal{B}}\right\}$. One can also expand $\left|\chi_{\mathcal{A}}\right\rangle$ and $\left|\chi_{\mathcal{B}}\right\rangle$ in terms of the corresponding Schmidt basis, given by

$$
\left|\chi_{\mathcal{A}}\right\rangle=\sum_{i} a_{i}\left|\mu_{\mathcal{A}}^{i}\right\rangle
$$

and

$$
\left|\chi_{\mathcal{B}}\right\rangle=\sum_{i} b_{i}\left|\nu_{\mathcal{B}}^{i}\right\rangle
$$

where $\sum_{i}\left|a_{i}\right|^{2}=1$ and $\sum_{i}\left|b_{i}\right|^{2}=1$. Using Eqs. (3) - (5), we can rewrite the second term in the right hand side of Eq. (1) as

$$
\begin{aligned}
\max _{|\chi\rangle \in n G}\left|\left\langle\chi \mid \psi_{12 \ldots N}\right\rangle\right| & =\max _{\left\{a_{i}\right\},\left\{b_{j}\right\}}\left|\sum_{i} \sum_{j} a_{i}^{*} b_{j}^{*}\left\langle\mu_{\mathcal{A}}^{i}\left|\otimes\left\langle\nu_{\mathcal{B}}^{j}\left|\sum_{k} \sqrt{\lambda_{k}}\right| \mu_{\mathcal{A}}^{k}\right\rangle \otimes\right| \nu_{\mathcal{B}}^{k}\right\rangle\right| \\
& =\max _{\left\{a_{i}\right\},\left\{b_{j}\right\}}\left|\sum_{k} a_{k}^{*} b_{k}^{*} \sqrt{\lambda_{k}}\right| \\
& \leq \max _{\left\{a_{i}\right\},\left\{b_{j}\right\}} \sum_{k}\left|a_{k}\right|\left|b_{k}\right| \sqrt{\lambda_{k}} .
\end{aligned}
$$

To obtain the inequality (7), we use the triangle inequal$i^{2}{ }^{2}$. The optimization over all nongenuinely multimode entangled states $|\chi\rangle$ now reduces to the optimization over the state parameters, $\left\{a_{i}\right\}$ and $\left\{b_{j}\right\}$. If we assume that the Schmidt coefficients $\left\{\lambda_{i}\right\}_{\mathrm{s}}$ are arranged in the descending order, we have

$$
\begin{aligned}
\max _{|\chi\rangle \in n G}\left|\left\langle\chi \mid \psi_{12 \ldots N}\right\rangle\right| & \leq \sqrt{\lambda_{1}} \max _{\left\{a_{i}\right\},\left\{b_{j}\right\}} \sum_{k}\left|a_{k}\right|\left|b_{k}\right| \\
& \leq \sqrt{\lambda_{1}} \max _{\left\{a_{i}\right\},\left\{b_{j}\right\}} \sqrt{\sum_{i}\left|a_{i}\right|^{2} \sum_{j}\left|b_{j}\right|^{2}} \\
& \leq \sqrt{\lambda_{1}} .
\end{aligned}
$$

The second inequality is obtained by using the well known Cauchy-Schwarz inequality, and the inequality (8) is due to the normalization conditions in terms of $a_{i}$ and $b_{j}$. By choosing $\left|a_{1}\right|=\left|b_{1}\right|=1$ and the rest of the coefficients to be 0 in Eq. (6), the above bound can be achieved, and hence the GGM of $\left|\psi_{12 \ldots N}\right\rangle$ reduces to

$$
\begin{aligned}
& \mathcal{G}\left(\left|\psi_{12 \ldots N}\right\rangle\right)= \\
& 1-\max \left\{\lambda_{\mathcal{A}: \mathcal{B}} \mid \mathcal{A} \cup \mathcal{B}=\{1,2, \ldots, N\}, \mathcal{A} \cap \mathcal{B}=\emptyset\right\},
\end{aligned}
$$

where $\lambda_{\mathcal{A}: \mathcal{B}}$ is the maximal Schmidt coefficient in the $\mathcal{A}: \mathcal{B}$ modal split of $\left|\psi_{12 \ldots N}\right\rangle$, and maximization is performed over all such possible mode-bipartitions. Equipping ourselves with the canonical formula of GGM, we

\footnotetext{
${ }^{2}|a+b| \leq|a|+|b|$.
}

now proceed to compute the GGM for pure multimode Gaussian states in terms of the symplectic invariants.

\section{GGM FOR PURE MULTIMODE GAUSSIAN STATES}

The covariance matrix of an arbitrary $m$-mode Gaussian state, $\rho$, is a $2 m \times 2 m$ matrix, $\Lambda$, defined by

$$
\Lambda_{i j}=\frac{1}{2}\left\langle\left\{R_{i}, R_{j}\right\}\right\rangle-\left\langle R_{i}\right\rangle\left\langle R_{j}\right\rangle,
$$

where $\vec{R}=\left(q_{1}, p_{1}, q_{2}, p_{2}, \ldots q_{m}, p_{m}\right)^{\mathrm{T}}$ with $q_{i}$ s and $p_{i}$ s being the usual quadrature operators. The quadrature operators are related to the field operators, $a_{i} \mathrm{~s}$, in the following way:

$$
q_{j}=\frac{1}{\sqrt{2}}\left(a_{j}+a_{j}^{\dagger}\right), p_{j}=\frac{1}{\sqrt{2} i}\left(a_{j}-a_{j}^{\dagger}\right),
$$

where $i=\sqrt{-1}$. The positivity of $\rho$ can be certified from the bonafied condition on the covariance matrix

$$
\Lambda+i J \geq 0, \text { where } J=\bigoplus_{i=1}^{m}\left[\begin{array}{cc}
0 & 1 \\
-1 & 0
\end{array}\right] .
$$

Here $J$ is the symplectic matrix. Following Williamson's theorem [48, 73], we notice that the covariance matrix $\Lambda$ can be obtained from $\Lambda^{d}$ by appropriate symplectic transformation $\left(\mathbf{S}_{\Lambda}\right)$,

$$
\Lambda=\mathbf{S}_{\Lambda} \Lambda^{d} \mathbf{S}_{\Lambda}^{\mathrm{T}}
$$




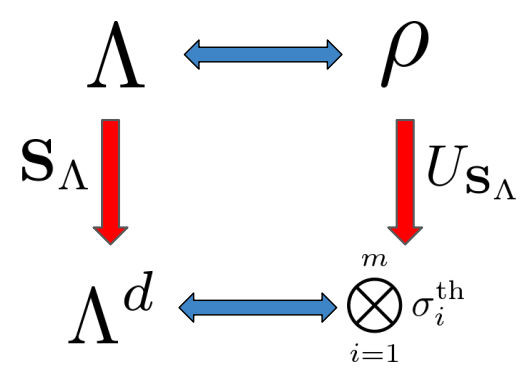

FIG. 1: Schematic representation of symplectic diagonalization. Any arbitrary Gaussian state can be written as a product of thermal states obtained by applying the appropriate unitary operation.

with

$$
\Lambda^{d}=\left[\begin{array}{lllll}
\nu_{1} \mathbb{I}_{2} & & & \\
& \nu_{2} \mathbb{I}_{2} & & \\
& & \cdot & \\
& & & \cdot & \\
& & & \nu_{m} \mathbb{I}_{2}
\end{array}\right]=\bigoplus_{i=1}^{m} \nu_{i} \mathbb{I}_{2},
$$

where $\left\{\nu_{i}\right\}_{\mathrm{s}}$ are the symplectic eigenvalues of $\Lambda$ and $\mathbb{I}_{2}$ is the $2 \times 2$ identity matrix. Note that $\Lambda^{d}$ corresponds to a product of thermal states

$$
\rho^{d}=\bigotimes_{i=1}^{m} \sigma_{\beta_{i}}, \text { with temperatures } \beta_{i}=\ln \frac{\nu_{i}+1 / 2}{\nu_{i}-1 / 2} \text {. }
$$

The state $\rho$ is related to $\rho^{d}$ via the relation $\rho=$ $U_{\mathbf{S}_{\Lambda}} \rho^{d} U_{\mathbf{S}_{\Lambda}}^{\dagger}$, where $U_{\mathbf{S}_{\Lambda}}$ is the unitary operator corresponding to the symplectic transform $\mathbf{S}_{\Lambda}$. Note that since $U_{\mathbf{S}_{\Lambda}}$ is unitary, the eigenspectrum of $\rho$ and $\rho^{d}$ are identical. The above analysis is schematically depicted in Fig. 1. In general, the eigenvalues, $\lambda_{n}$, of a thermal state with inverse temperature $\beta$ is given by $\lambda_{n}=e^{-\beta n}\left(1-e^{-\beta}\right)$. The maximal eigenvalue simply corresponds to $\lambda_{n=0}$. Now, the eigenvalues of $\rho^{d}$, which are also eigenvalues of $\rho$ are then given by

$$
\lambda_{n_{1}, n_{2}, \ldots n_{m}}=\prod_{i} \lambda_{n_{i}} .
$$

The maximal eigenvalue of $\rho$ is therefore given by

$$
\lambda_{n_{1}=0, n_{2}=0, \ldots n_{m}=0}=\prod_{i=1}^{m}\left(1-e^{-\beta_{i}}\right)=\prod_{i=1}^{m} \frac{2}{1+2 \nu_{i}},
$$

where $\nu_{i}$ is the symplectic eigenvalue of the $i^{\text {th }}$ mode. Expressing the maximal eigenvalue in terms of the symplectic specra enables evaluation of GGM in a much more efficient way which we encapsulate below in the form of the following theorem:

Theorem 1. The GGM (G) of a $N$-mode pure Gaussian state $\left|\psi_{12 \ldots N}\right\rangle$ is given by

$$
\mathcal{G}\left(\left|\psi_{12 \ldots N}\right\rangle\right)=1-\max \mathcal{P}_{m}\left\{\prod_{i=1}^{m} \frac{2}{1+2 \nu_{i}}\right\}_{m=1}^{\left[\frac{N}{2}\right]},
$$

where $\mathcal{P}_{m}$ denotes all the reduced states of $|\psi\rangle_{12 \ldots N}$ with $m$-modes, and $[x]$ denotes the integral part of $x$.

Proof. The canonical formula for the GGM in Eq. (9) reveals that the computation of GGM is equivalent to the evaluation of the maximum eigenvalue of all the reduced states of the given state $\left|\psi_{12 \ldots N}\right\rangle$. The canonical formula can be restated as

$$
\mathcal{G}\left(\left|\psi_{12 \ldots N}\right\rangle\right)=1-\max \mathcal{P}_{m}\left\{\lambda_{n_{1}, n_{2}, \ldots n_{m}}\right\}_{m=1}^{\left[\frac{N}{2}\right]},
$$

where $\lambda_{n_{1}, n_{2}, \ldots n_{m}}$ s denote the eigenvalues of a particular $m$-mode reduced state of $\left|\psi_{12 \ldots N}\right\rangle$, and $\mathcal{P}_{m}$ denotes all the reduced states of $\left|\psi_{12 \ldots N}\right\rangle$ with $m$-modes.

From Eq. (17), we know that for a particular $m$-mode reduced state with spectra $\nu_{i} \mathrm{~s}$, the maximal eigenvalue simply reads $\prod_{i=1}^{m} \frac{2}{1+2 \nu_{i}}$. The formula of GGM is then obtained by maximizing the maximal eigenvalue of all the $m \in[1,[N / 2]]$-mode reductions of $|\psi\rangle_{12 \ldots N}$, and therefore we have

$$
\mathcal{G}\left(\left|\psi_{12 \ldots N}\right\rangle\right)=1-\max \mathcal{P}_{m}\left\{\prod_{i=1}^{m} \frac{2}{1+2 \nu_{i}}\right\}_{m=1}^{\left[\frac{N}{2}\right]},
$$

and hence the proof.

We, therefore, arrive at our goal of expressing the GGM of a pure multimode Gaussian state in terms of the symplectic spectrum of its reduced states. Below, we provide a prescription for computing the GGM for an arbitrary multimode pure state $\left|\psi_{12 \ldots N}\right\rangle$ in terms of the following steps which illustrates the simplicity of the method:

1. Evaluate all the $m$-mode reduced density matrices, $\rho_{m}$ of $\left|\psi_{12 \ldots N}\right\rangle$, where $m \in\left[1,\left[\frac{N}{2}\right]\right]$. Note that there are $\left(\begin{array}{l}N \\ m\end{array}\right)$ different $m$-mode reductions of the initial $N$-mode state.

2. Perform symplectic diagonalization of all the $\rho_{m} \mathrm{~s}$ to get the diagonal $\rho_{m}^{d} \mathrm{~s}$ and the corresponding symplectic eigenvalues, $\nu_{i}^{m} \mathrm{~s}$, of each $\rho_{m}$.

3. Compute the maximal eigenvalue, $\bar{\lambda}_{m}^{\max }$, of each $\rho_{m}$ using the formula

$$
\bar{\lambda}_{m}^{\max }=\prod_{i=1}^{m} \frac{2}{1+2 \nu_{i}} .
$$

Choose the maximum eigenvalue $\lambda_{m}^{\max }$ out of all the $\bar{\lambda}_{m}^{\max } \mathrm{s}$.

4. The GGM of $|\psi\rangle_{1,2, \ldots N}$ is then calculated from the following expression

$$
\mathcal{G}\left(\left|\psi_{12 \ldots N}\right\rangle\right)=1-\max \left\{\lambda_{m}^{\max }\right\}_{m=1}^{\left[\frac{N}{2}\right]} .
$$

We want to highlight here that the evaluation of GGM for multimode Gaussian states is much simpler compared to that for a multiqubit state. This is so because the 
$m$-mode reductions in case of Gaussian states are characterized by $2 m \times 2 m$ covariance matrices while the $m$ party reduced states for a multiqubit state is a $2^{m} \times 2^{m}$ dimensional density matrix. This difference in computational complexity (polynomial vs. exponential) implies that via exact diagonalization, we can atmost compute GGM of a $\sim 24$-qubit state, whereas we can go, at least in principle, upto $2^{12}$-modes. Ultimately this number would be restricted by the number of reduced density matrices, $\left(\begin{array}{l}N \\ m\end{array}\right)$, for which we have to compute the eigenvalues for every $m$-mode reductions of the given $N$-mode pure state. This fact remains true in the multiqubit case also. Furthermore, partial tracing at the covariance matrix level is much easier compared to partial tracing at the level of states. Hence, our analysis provides an efficiently computable and scalable method to calculate the genuine multimode entanglment content of the Gaussian states.

As an aside, we want to recall that GGM is a measure of geometric origin which, as mentioned earlier, is defined as the minimum distance of the given state (whose genuine multiparty entanglement content has to be calculated) from the set of non-genuinely multiparty entangled states. Hence, by definition, it is applicable for all states, both pure and mixed. However, for pure states, owing to Schmidt decomposition, the minimization problem becomes tractable and we have the canonical formula for GGM. In the case of mixed states, such simplification is unavailable. As shown for finite dimensional systems, if the states have some symmetries [47, 74], the optimization can "sometimes" be performed. Note that even for pure states, computation of GGM becomes challenging with the increase in the number of parties and their dimensions, let alone when the dimension of each party becomes infinite, the case considered in our work. This is due to the exponential growth of the size and number of partitions one has to diagonalize to compute GGM.

Nevertheless, in the next section, we use the above described prescription to compute the patterns of GGM for some typical Gaussian states and illustrate their multimode entanglement numerically.

\section{A. GGM of some typical Gaussian states}

We start our analysis with a three-mode pure Gaussian state. The first example is a three-mode state prepared by combining three single-mode squeezed states in a tritter (a three-mode generalization of a beam-splitter) [73]. Secondly, we track the dynamics of GGM of states generated in a single nonlinear crystal. Next we consider the case of how well can bimodal entanglement be distributed among three modes by means of passive operations using beam splitters. In the genre of four modes, we calculate the GGM of the four-mode squeezed vaccum with respect to the squeezing parameter.

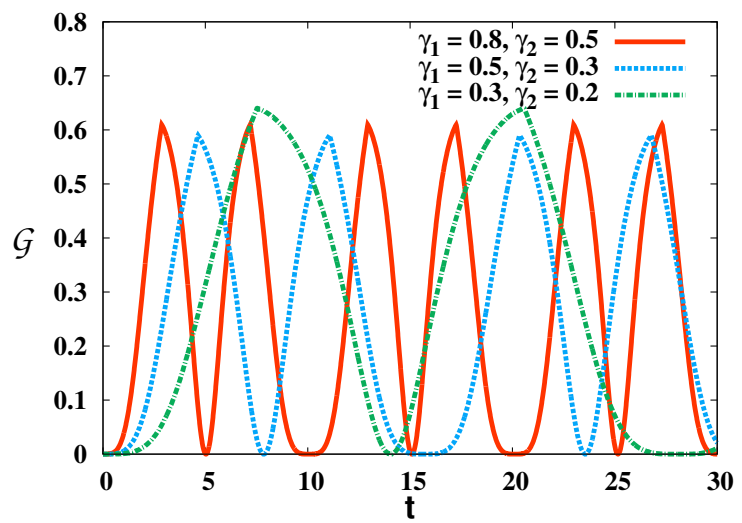

FIG. 2: (Color online) GGM of $\left|\psi_{3}^{b}(t)\right\rangle$ vs. time. The state is generated according to the Hamiltonian $H_{I}$, given in Eq. (27). Solid, dotted and dashed lines are for different choices of $\gamma_{1}$ and $\gamma_{2}$ values.

\section{Three-mode Gaussian states}

Example 1. Three single-mode squeezed vacuum states of strength $r$ combined using the "tritter" (a three-mode generalization of the beam splitter) gives a three-mode state, $\left|\psi_{3}^{a}\right\rangle$, which possess the covariance matrix, given by $[73]$

$$
\Lambda^{a}=\frac{1}{2}\left[\begin{array}{cccccc}
\mathcal{R}_{+} & 0 & \mathcal{S} & 0 & \mathcal{S} & 0 \\
0 & \mathcal{R}_{-} & 0 & -\mathcal{S} & 0 & -\mathcal{S} \\
\mathcal{S} & 0 & \mathcal{R}_{+} & 0 & \mathcal{S} & 0 \\
0 & -\mathcal{S} & 0 & \mathcal{R}_{-} & 0 & -\mathcal{S} \\
\mathcal{S} & 0 & \mathcal{S} & 0 & \mathcal{R}_{+} & 0 \\
0 & -\mathcal{S} & 0 & -\mathcal{S} & 0 & \mathcal{R}_{-}
\end{array}\right]
$$

where $\mathcal{R}_{ \pm}=\cosh 2 r \pm \frac{1}{3} \sinh 2 r$ and $\mathcal{S}=-\frac{2}{3} \sinh 2 r$. It turns out to be a symmetric state with identical reduced single-mode covariance matrices $\left(\Lambda_{1}^{a}=\Lambda_{2}^{a}=\Lambda_{3}^{a}\right)$, which reads as

$$
\Lambda_{1}^{a}=\frac{1}{2}\left[\begin{array}{cc}
\mathcal{R}_{+} & 0 \\
0 & \mathcal{R}_{-}
\end{array}\right]
$$

Following the prescription for calculating the GGM, we note that for three-mode states, we only need to consider the single-mode reductions $\left(\left[\frac{3}{2}\right]=1\right)$. The symplectic eigenvalues of $\Lambda_{1}^{a}$ is given by

$$
\nu_{1}=\frac{1}{2} \sqrt{\mathcal{R}_{+} \mathcal{R}_{-}}=\frac{1}{6} \sqrt{5+4 \cosh 4 r},
$$

leading to GGM as

$$
\mathcal{G}\left(\left|\psi_{3}^{a}\right\rangle\right)=1-\frac{2}{1+2 \nu_{1}}=\frac{\frac{1}{3} \sqrt{5+4 \cosh 4 r}-1}{\frac{1}{3} \sqrt{5+4 \cosh 4 r}+1} .
$$

We, therefore, obtain an expression of GGM as a function of the squeezing strength $r$. Expectedly, as $r \rightarrow \infty$, the GGM approaches its algebraic maximum of unity. 
Example 2. Let us consider a three-mode vacuum state induced by an interaction Hamiltonian, $H_{I}$, describing action of a single nonlinear crystal

$$
H_{I}=\gamma_{1} a_{1}^{\dagger} a_{3}^{\dagger}+\gamma_{2} a_{2}^{\dagger} a_{3}+\text { h.c. }
$$

where $a_{i}^{\dagger}$ and $a_{i}$ represent the creation and the anhilation operators respectively. The effective coupling constants $\gamma_{k}, k=1,2$, are functions of the nonlinear susceptibilities and the pump intensities. The Hamiltonian $H_{I}$ describes interactions among three modes of the radiation field which are coupled via two parametric pumps. Such a process can also be realized exprimentally in $\chi^{(2)}$ media [75]. We consider the three-mode vacuum state as the initial state and then the system evolves according to the Hamiltonian $H_{I}$, i.e. the time evolved threemode state at any given time, $t$, can be represented as $\left|\psi_{3}^{b}(t)\right\rangle=e^{-i H_{I} t}|0\rangle \otimes|0\rangle \otimes|0\rangle$. The closed form expression of the same can also be computed $[73,75]$ as

$$
\left|\psi_{3}^{b}(t)\right\rangle=\frac{1}{\sqrt{1+n_{1}}} \sum_{r, s=0}^{\infty}\left(\frac{n_{2}}{1+n_{1}}\right)^{r / 2}\left(\frac{n_{3}}{1+n_{1}}\right)^{s / 2} e^{-i\left(r \phi_{2}+s \phi_{3}\right)} \sqrt{\frac{(r+s) !}{r ! s !}}|r+s, r, s\rangle,
$$

where $n_{i}=\left\langle a_{i}^{\dagger} a_{i}\right\rangle$ denotes the average number of photons in the $i$-th mode with $\phi_{j}$ s being the phase factors. For this Hamiltonian, we have $n_{1}=n_{2}+n_{3}$ at all times with

$$
\begin{aligned}
& n_{2}=\frac{\left|\gamma_{1}\right|^{2}\left|\gamma_{2}\right|^{2}}{\Omega^{4}}(1-\cos \Omega t)^{2}, \\
& n_{3}=\frac{\left|\gamma_{1}\right|^{2}}{\Omega^{2}} \sin ^{2} \Omega t
\end{aligned}
$$

where $\Omega=\sqrt{\left|\gamma_{2}\right|^{2}-\left|\gamma_{1}\right|^{2}}$. The covariance matrix, $\Lambda^{b}$, of the above state [73] is

$$
\Lambda^{b}=\left[\begin{array}{cccccc}
\mathcal{F}_{1} & 0 & \mathcal{A}_{2} & -\mathcal{B}_{2} & \mathcal{A}_{3} & -\mathcal{B}_{3} \\
0 & \mathcal{F}_{1} & -\mathcal{B}_{2} & -\mathcal{A}_{2} & -\mathcal{B}_{3} & -\mathcal{A}_{3} \\
\mathcal{A}_{2} & -\mathcal{B}_{2} & \mathcal{F}_{2} & 0 & \mathcal{C} & \mathcal{D} \\
-\mathcal{B}_{2} & -\mathcal{A}_{2} & 0 & \mathcal{F}_{2} & -\mathcal{D} & \mathcal{C} \\
\mathcal{A}_{3} & -\mathcal{B}_{3} & \mathcal{C} & -\mathcal{D} & \mathcal{F}_{3} & 0 \\
-\mathcal{B}_{3} & -\mathcal{A}_{3} & \mathcal{D} & \mathcal{C} & 0 & \mathcal{F}_{3}
\end{array}\right]
$$

where $\mathcal{F}_{i}=n_{i}+1 / 2, \mathcal{A}_{i}=\sqrt{n_{i}\left(1+n_{1}\right)} \cos \phi_{i}, \mathcal{B}_{i}=$ $\sqrt{n_{i}\left(1+n_{1}\right)} \sin \phi_{i}, \mathcal{C}=\sqrt{n_{2} n_{3}} \cos \left(\phi_{2}-\phi_{3}\right)$, and $\mathcal{D}=$ $\sqrt{n_{2} n_{3}} \sin \left(\phi_{2}-\phi_{3}\right)$. The single-mode reduced covariance matrices $\Lambda_{i}^{b} \mathrm{~s}$ in this case are

$$
\Lambda_{i}^{b}=\mathcal{F}_{i}\left[\begin{array}{ll}
1 & 0 \\
0 & 1
\end{array}\right] .
$$

Notice that unlike in the previous case with $\left|\psi_{3}^{a}\right\rangle$, the single-mode reduced covariance matrices, $\Lambda_{i}^{b} \mathrm{~s}$, are not identical. Nevertheless, all of them come in diagonalized form with symplectic eigenvalues, $\left\{\mathcal{F}_{1}, \mathcal{F}_{2}, \mathcal{F}_{3}\right\}$. Hence the GGM reads as

$$
\mathcal{G}\left(\left|\psi_{3}^{b}(t)\right\rangle\right)=1-\max \left\{\frac{2}{1+2 \mathcal{F}_{i}}\right\}_{i=1}^{3},
$$

where $\mathcal{F}_{i}$ s are functions of time. For fixed $\gamma_{i}$ s, the oscillatory behavior of GGM for the evolved state against time is depicted in Fig. 2. Note that the output state is independent of phase factors. The presence of kinks (nonanalyticities) in the dynamics is due to the maximization present in the formula of GGM. The times at which these kinks appear are precisely those where there is a crossover among the first two highest eigenvalues, specifically, when the second highest eigenvalue becomes the maximal one.

\section{Four-mode Gaussian states}

Let us now move to a computation of genuine multimode entanglement of a four-mode Gaussian state, namely the four-mode squeezed vacuum (FMSV) state [66]. The FMSV state can be obtained by using two single-mode squeezed vacuum passing through a 50:50 beam splitter followed by another two beam splitter on the output modes of the previous one. The FMSV state can be represented as

$$
\begin{aligned}
|F M S V\rangle= & e^{\frac{r}{2} \sum_{i=1}^{4} a_{i}^{\dagger} a_{i+1}^{\dagger}-a_{i} a_{i+1}}|0000\rangle \\
= & \frac{1}{\cosh r} \sum_{n=0}^{\infty} \sum_{r_{1}=0}^{n} \sum_{r_{2}=0}^{n} \sqrt{\left(\begin{array}{c}
n \\
r_{1}
\end{array}\right)} \sqrt{\left(\begin{array}{c}
n \\
r_{2}
\end{array}\right)} \\
& \left(\frac{1}{2} \tanh r\right)^{n}\left|n-r_{1}\right\rangle\left|n-r_{2}\right\rangle\left|r_{1}\right\rangle\left|r_{2}\right\rangle,
\end{aligned}
$$

where for $i=4, i+1$ is considered to be 1 . It is clear from the above expression that the FMSV state is translationally invariant with the interchange of mode $1 \leftrightarrow 3$ and $2 \leftrightarrow 4$. Hence, we call modes $(1,2)$ and $(1,4)$ as adjacent modes while $(1,3)$ and $(2,4)$ as alternate modes.

While computing GGM, the above symmetries greatly simplify the evaluation by reducing the search space during maximization. In particular, we have to compute eigenvalues of the reduced covariance matrices of a single-mode, $\Lambda_{\text {single }}^{\mathrm{FMSV}}$, and two-mode reduced states consisting of adjacent and alternate modes, denoted respectively by $\Lambda_{\text {adjacent }}^{\mathrm{FMSV}}$ and $\Lambda_{\text {alternate }}^{\mathrm{FMSV}}$ For brevity, we do not give the expressions of the covariance ma- 
trix here (see Appendix B for their forms), $\Lambda^{\mathrm{FMSV}}$, and its relevant reduced covariance matrices, $\Lambda_{\text {single }}^{\mathrm{FMSV}}$, $\Lambda_{\text {adjacent }}^{\mathrm{FMSV}}$, and $\Lambda_{\text {alternate }}^{\mathrm{FMSV}}$ Nevertheless, the symplectic eigenvalue of $\Lambda_{\text {single }}^{F M S V}$ is computed to be $\frac{1}{2} \cosh ^{2} r$, while those of $\Lambda_{\text {adjacent }}^{F M S V}$ and $\Lambda_{\text {alternate }}^{F M S V}$ are $\left\{\frac{1}{2} \cosh r, \frac{1}{2} \cosh r\right\}$ and $\left\{\frac{1}{2}, \frac{1}{2} \cosh 2 r\right\}$ respectively. Therefore, the maximal eigenvalues from each of these sectors read as $\left\{\frac{2}{1+\cosh ^{2} r}, \frac{2}{1+\cosh 2 r},\left(\frac{2}{1+\cosh r}\right)^{2}\right\}$, and hence the GGM of FMSV state can be expressed as

$$
\mathcal{G}(|F M S V\rangle)=1-\max \left\{\frac{2}{1+\cosh ^{2} r}, \frac{2}{1+\cosh 2 r},\left(\frac{2}{1+\cosh r}\right)^{2}\right\} .
$$

As expected, when the squeezing strength $r$ takes infinitely large values, the GGM of FMSV approaches unity.

\section{GGM OF NON-GAUSSIAN STATES}

As mentioned before, non-Gaussian resources can outperform their classical counterparts in a variety of quantum information and computation protocols. Therefore, we now venture into the non-Gaussian regime and examine whether it offers any enhancement of GGM. Moreover, as already discussed, we choose to de-Gaussify states by photon addition and subtraction for their easy and scalable experimental implementability. Note that such incremental behaviour on addition (subtraction) of photons was reported in the case of bipartite non-classical correlations for two and four-mode states [65, 66, 7679]. These results motivate us to consider the possibility of enhancement of genuine multimode entanglement on adding or subtracting photons from Gaussian states.

In the preceding section, we calculated GGM for several classes of three-mode and four-mode Gaussian states by using the form in terms of the symplectic eigenvalues of the covariance matrices derived in Eq. (18) of Theorem 1. However, such simplification does not work, when one adds (subtracts) photons in (from) Gaussian states. This is due to the fact that the photon-added (-subtracted) states do not represent Gaussian states and hence cannot be completely described by covariance matrices. In this section, we use the cannonical form of GGM given in Eq. (9) for the non-Gaussian states. Our aim is to characterize GGM for several classes of non-Gaussian states, emerging from the three-mode state (given in Eq. (28)), and the FMSV state, by adding and subtracting photons.

It is worth mentioning that by the term photon addition and subtraction in different mode(s), we mean a consecutive addition in or subtraction of photons from that particular mode(s). It is indeed true that non-Gaussian states can also be obtained by several mixed ordering of photon addition and subtraction. But we will not be considering such cases here.

Another important aspect of this photon addition and subtraction operation is that it is a physical operation

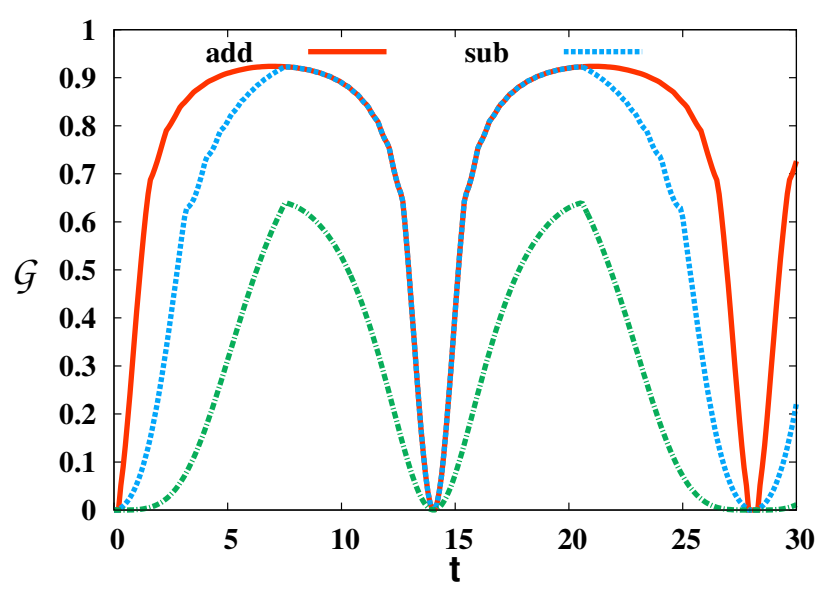

FIG. 3: GGM of the Gaussian as well as non-Gaussian states against time. In particular, we consider a three-mode Gaussian state $\left|\psi_{3}^{b}(t)\right\rangle$ (green dot-dashed) and non-Gaussian states, $\left|\psi_{3}^{b}(t)^{a d d\left\{m_{i}\right\}}\right\rangle$ (red solid) and $\left|\psi_{3}^{b}(t)^{s u b\left\{m_{i}\right\}}\right\rangle$ (blue dashed), given in Eqs. (35) and (36). Here $\gamma_{1}=0.8$ and $\gamma_{2}=0.5$. In the construction of non-Gaussian states $\left|\psi_{3}^{b}(t)^{\text {add }\left\{m_{i}\right\}}\right\rangle$ and $\left|\psi_{3}^{b}(t)^{s u b\left\{m_{i}\right\}}\right\rangle$, we choose $m_{1}=5, m_{2}=0$ and $m_{3}=0$.

on the given quantum state, based on the post-selection, i.e., it is a non-unitary operation. Here our main objective is to study genuine multimode entanglement content in these non-Gaussian states, where addition and subtraction of photons has been used as a tool to generate them. We then compare the GGM of photon-added (subtracted) state with the GGM of the initial Gaussian state and address the question whether addition is better than subtraction from the perspective of amount of genuine multimode entanglement in these states.

\section{A. Three-mode non-Gaussian state}

Let us first consider the non-Gaussian states derived from the three-mode Gaussian states, given in Eq. (28), which are de-Gaussified via addition or subtraction of photons. The state takes the following form after adding $m_{i}(i=1,2,3)$ number of photons in mode $i$, denoted by 
$\left|\psi_{3}^{b}(t)^{a d d\left\{m_{i}\right\}}\right\rangle$ and similarly the photon-subtracted state, $\quad\left|\psi_{3}^{b}(t)^{s u b\left\{m_{i}\right\}}\right\rangle$ :

$$
\begin{aligned}
\left|\psi_{3}^{b}(t)^{a d d\left\{m_{i}\right\}}\right\rangle=\frac{1}{\sqrt{N^{a d d}}} \sum_{r, s=0}^{\infty}\left(\frac{n_{2}}{1+n_{1}}\right)^{r / 2}\left(\frac{n_{3}}{1+n_{1}}\right)^{s / 2} e^{-i\left(r \phi_{2}+s \phi_{3}\right)} \sqrt{\frac{(r+s) !}{r ! s !}} \sqrt{\frac{\left(r+s+m_{1}\right) !}{(r+s) !}} \\
\left.\left.\sqrt{\frac{\left(r+m_{2}\right) !}{r !}} \sqrt{\frac{\left(s+m_{3}\right) !}{s !}}\left|r+s+m_{1}\right\rangle r+m_{2}\right\rangle s+m_{3}\right\rangle, \\
\left|\psi_{3}^{b}(t)^{s u b\left\{m_{i}\right\}}\right\rangle=\frac{1}{\sqrt{N^{s u b}}} \sum_{\substack{r=m_{2}, s=m_{3} \\
m_{1} \geq m_{2}+m_{3}}}^{\infty}\left(\frac{n_{2}}{1+n_{1}}\right)^{r / 2}\left(\frac{n_{3}}{1+n_{1}}\right)^{s / 2} e^{-i\left(r \phi_{2}+s \phi_{3}\right)} \sqrt{\frac{(r+s) !}{r ! s !}} \sqrt{\frac{(r+s) !}{\left(r+s-m_{1}\right) !}} \\
\left.\left.\sqrt{\frac{r !}{\left(r-m_{2}\right) !}} \sqrt{\frac{s !}{\left(s-m_{3}\right) !}}\left|r+s-m_{1}\right\rangle r-m_{2}\right\rangle s-m_{3}\right\rangle,
\end{aligned}
$$

where $N^{a d d}$ and $N^{s u b}$ are the normalization constants of the photon-added and -subtracted states. $\mathcal{G}\left(\left|\psi_{3}^{b}(t)\right\rangle^{\text {add }\left\{m_{i}\right\}}\right)$ (the purple line) and $\mathcal{G}\left(\left|\psi_{3}^{b}(t)\right\rangle^{\text {sub }\left\{m_{i}\right\}}\right.$ ) (the green line) are plotted in Fig. 3, where we choose $m_{1}=5$ and $m_{2}=m_{3}=0$, i.e., 5 photons are added (subtracted) in (from) the first mode and no photons are added and subtracted from the rest of the modes. We observe that although the oscillatory behavior of GGM remains same, there is an enhancement of genuine multimode entanglement for both the non-Gaussian states (given in Eqs (35) and (36)), compared to their Gaussian counterpart, for all values of $t$. Moreover, we find that each oscillation contains two humps - the first one is the mirror image of the second one. Specifically, we find that, in the first hump, GGM increases with $t$, and $\mathcal{G}\left(\left|\psi_{3}^{b}(t)\right\rangle^{a d d\left\{m_{i}\right\}}\right) \geq \mathcal{G}\left(\left|\psi_{3}^{b}(t)\right\rangle^{\operatorname{sub}\left\{m_{i}\right\}}\right)$, thereby showing advantage of adding photons over subtraction. After $\mathcal{G}\left(\left|\psi_{3}^{b}(t)\right\rangle^{a d d}\left\{m_{i}\right\}\right)$ and $\mathcal{G}\left(\left|\psi_{3}^{b}(t)\right\rangle^{s u b\left\{m_{i}\right\}}\right)$ reach their maxima, they coincide and start decreasing together with time. The behavior in the second hump images that of the first one. This example shows that by proper tuning of interaction strengths, photon-addition can enhance genuine multimode entanglement upto $\sim 30 \%$ (as seen in Fig. 3). All the curves, obtained both from Gaussian and non-Gaussian states coincide at those times when $\mathcal{G}=0$. This is because in the first hump, bare Gaussian state itself becomes product at that time and we cannot generate multimode entangled states by adding or subtracting photons.

\section{B. GGM of non-Gaussian states originated from FMSV}

We now investigate the multimode entanglement content of a four-mode non-Gaussian state which emerges by adding and subtracting photons from the modes of the FMSV state given in Eq. (33), and our aim is to find
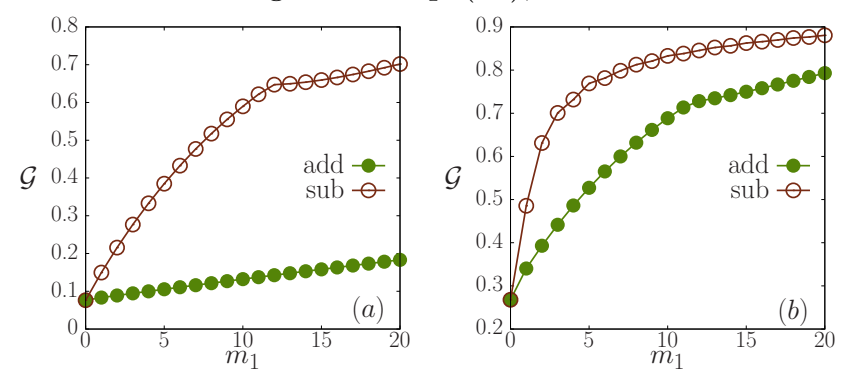

FIG. 4: (Color online) GGM against added (solid circles) and subtracted (hollow circles) photons from the first mode, $m_{1}$. In (a), we set the squeezing strength, $\mathrm{r}=0.4$, while we choose $r=0.8$ in (b). In both the cases, subtraction yields higher value of GGM than that of the addition.

whether the increase of multimode entanglement with respect to addition and subtraction of photons as seen in three-mode non-Gaussian states can also persist in the four-mode case also. The photon-added and -subtracted FMSV states read respectively as 


$$
\begin{gathered}
\left|\psi_{F M S V}^{\text {add }\left\{m_{i}\right\}}\right\rangle=\frac{1}{N^{a d d}} \sum_{n=0}^{\infty} \sum_{r_{1}=0}^{n} \sum_{r_{2}=0}^{n}\left(\frac{1}{2} \tanh r\right)^{n} \sqrt{\left(\begin{array}{c}
n \\
r_{1}
\end{array}\right)} \sqrt{\left(\begin{array}{c}
n \\
r_{2}
\end{array}\right)} \sqrt{\frac{\left(n-r_{1}+m_{1}\right) !}{\left(n-r_{1}\right) !} \sqrt{\frac{\left(n-r_{2}+m_{2}\right) !}{\left(n-r_{2}\right) !}}} \\
\sqrt{\frac{\left(r_{1}+m_{3}\right) !}{r_{1} !}} \sqrt{\frac{\left(r_{2}+m_{4}\right) !}{r_{2} !}}\left|n-r_{1}+m_{1}\right\rangle\left|n-r_{2}+m_{2}\right\rangle\left|r_{1}+m_{3}\right\rangle\left|r_{2}+m_{4}\right\rangle, \\
\left|\psi_{F M S V}^{\text {sub }\left\{m_{i}\right\}}\right\rangle=\frac{1}{N^{s u b}} \sum_{n=M}^{\infty} \sum_{r_{1}=m_{3} r_{2}=m_{4}}^{n-m_{1}} \frac{n-m_{2}}{2} \operatorname{tanhr)^{n}\sqrt {(\begin{array} {c}
{n}\\
{r_{1}}
\end{array} )}} \sqrt{\left(\begin{array}{c}
n \\
r_{2}
\end{array}\right)} \sqrt{\frac{\left(n-r_{1}\right) !}{\left(n-r_{1}-m_{1}\right) !}} \sqrt{\frac{\left(n-r_{2}\right) !}{\left(n-r_{2}-m_{2}\right) !}} \\
\sqrt{\frac{r_{1} !}{\left(r_{1}-m_{3}\right) !}} \sqrt{\frac{r_{2} !}{\left(r_{2}-m_{4}\right) !}}\left|n-r_{1}-m_{1}\right\rangle\left|n-r_{2}-m_{2}\right\rangle\left|r_{1}-m_{3}\right\rangle\left|r_{2}-m_{4}\right\rangle,
\end{gathered}
$$

where we use the convention that $m_{i}$ number of photons are added and subtracted in or from the mode $i$ $(i=1,2,3,4)$. $\quad N^{a d d}$ and $N^{s u b}$ are the respective normalization constants and $M=\max \left\{m_{1}+m_{3}, m_{2}+m_{4}\right\}$. Below, we catalogue the response of GGM to the operations of photon addition and subtraction:

1. Single-mode operations: We find the pattern of GGM when we add or subtract photons from any one of the four modes, of the FMSV state. Note that in case of single-mode photon operation, behavior of GGM remains independent of the choice of the mode taken for the operation. This is so because of the translational invariance of the Gaussian FMSV state. Without loss of generality, we choose the first mode for the operation, i.e., $\mathcal{G}$ is computed by varying $m_{1}$, and $m_{2}=m_{3}=m_{4}=0$. The plot of $\mathcal{G}\left(\left|\psi_{F M S V}^{a d d\left\{m_{1}\right\}}\right\rangle\right)$ and $\mathcal{G}\left(\left|\psi_{F M S V}^{s u b\left\{m_{1}\right\}}\right\rangle\right)$, with respect to $m_{1}$ is depicted in Fig. 4, for two different values of the squeezing parameter $r=0.4$ (in Fig. $4(\mathrm{a})$ ) and $r=0.8$ (in Fig. 4(b)). From this figure, it is prominent that $\mathcal{G}\left(\left|\psi_{F M S V}^{s u b\left\{m_{1}\right\}}\right\rangle\right) \geq \mathcal{G}\left(\left|\psi_{F M S V}^{a d d\left\{m_{1}\right\}}\right\rangle\right)$ for fixed values of $m_{1}$, i.e., non-Gaussian state obtained by photon subtraction posses more genuine multimode entanglement than its photon-added counterpart, although both of them possess high amount of GGM compared to the corresponding Gaussian state (see $m_{1}=0$ point in Fig. 4). However, with the increase of the squeezing parameter, $r$ of the initial Gaussian state, the difference of GGM between the photon-subtracted and -added states diminishes due to the substantial increase of multimode entanglement in the photon-added state.

2. Two-mode operations: Let us now observe whether such increment of multimode entanglement over the Gaussian states occurs even when photon-addition (-subtraction) are performed in two modes. In this situation, we consider two scenarios -

(a) unconstrained (independent) mode operations
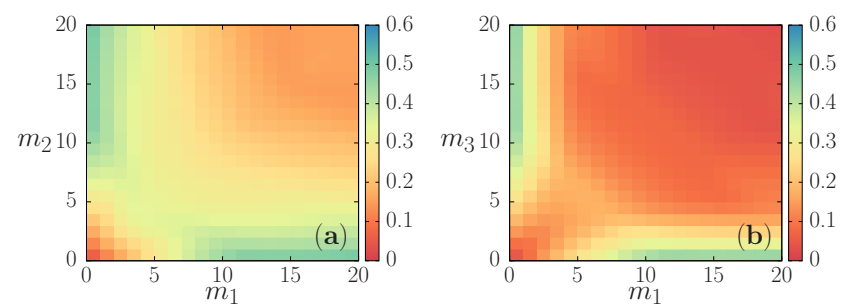

FIG. 5: Difference between GGM of the photon-subtracted and -added states against $\left(m_{1}, m_{2}\right)$-plane in (a) and $\left(m_{1}, m_{3}\right)$ plane in (b). In particular, we study the behavior of $\mathcal{G}\left(\left|\psi_{F M S V}^{s u b\left\{m_{1}, m_{2}\right\}}\right\rangle\right)-\mathcal{G}\left(\left|\psi_{F M S V}^{\text {add }\left\{m_{1}, m_{2}\right\}}\right\rangle\right)$, and $\mathcal{G}\left(\left|\psi_{F M S V}^{s u b\left\{m_{1}, m_{3}\right\}}\right\rangle\right)-$ $\mathcal{G}\left(\left|\psi_{F M S V}^{a d d}\left\{m_{1}, m_{3}\right\}\right\rangle\right)$. Positive values guarantee that subtraction is always better than that of the addition. We set $r=0.4$.

in which photons are added (subtracted) in (from) two modes independently.

(b) constrained mode operations where the total number of photons added (subtracted) in two modes are fixed.

By using the symmetry of the FMSV state, we obtain that there are only two choices of operations possible: (i) operations in the alternate modes and (ii) operations involving the adjacent modes. Therefore, all two-mode operations can be segregated into these two bins. Without any loss of generality, we refer to operations in modes 1 and 2 as adjacent and that between modes 1 and 3 being alternate. In our case, we fix the number of photons added or subtracted from mode 1 to be $m_{1}$, while that from modes 2 and 3 are both fixed to $n$, i.e., $m_{2}=m_{3}=n$, and the corresponding states would be denoted by $\left|\psi_{F M S V}^{a d d\left\{m_{1}, m_{2}=n\right\}}\right\rangle$ and $\left|\psi_{F M S V}^{s u b\left\{m_{1}, m_{2}=n\right\}}\right\rangle$ respectively.

- For unconstrained two-mode operations, which include both adjacent and alternate mode operations, photon-subtraction yields higher GGM com- 
pared to photon-addition. We observe this by considering the difference in GGM values of the two states, one of which is obtained by subtracting photons from the FMSV state, in the adjacent (alternate) modes, while the other by adding the same number of photons to the FMSV state in the adjacent (alternate) modes. Specifically, we observe that the positive value of the quantity $\mathcal{G}\left(\left|\psi_{F M S V}^{s u b\left\{m_{1}, m_{2}\right\}}\right\rangle\right)-\mathcal{G}\left(\left|\psi_{F M S V}^{a d d\left\{m_{1}, m_{2}\right\}}\right\rangle\right)$ in Fig. 5 (a), and $\mathcal{G}\left(\left|\psi_{F M S V}^{\text {sub }\left\{m_{1}, m_{3}\right\}}\right\rangle\right)-\mathcal{G}\left(\left|\psi_{F M S V}^{\text {add }\left\{m_{1}, m_{3}\right\}}\right\rangle\right)$ in Fig. 5 (b) ensure the superiority of photon-subtraction in these cases.

Let us now find whether operations in adjacent modes is more beneficial than that of the alternate modes. To address this question, we now restrict ourselves to photon addition. In this case, the operations in alternate modes gives higher value of GGM than that of the adjacent modes. We demonstrate this by plotting $\mathcal{G}\left(\left|\psi_{F M S V}^{a d d\left\{m_{1}, m_{3}=n\right\}}\right\rangle\right)-$ $\mathcal{G}\left(\left|\psi_{F M S V}^{a d d\left\{m_{1}, m_{2}=n\right\}}\right\rangle\right)$ in Fig. 6(a). The positive value in the entire ranges of $\left(m_{1}, n\right)$-plane certifies supremacy of alternate operations in case of photon addition. Interestingly, for photon subtraction, we observe a completely opposite effect, i.e., the adjacent operations lead to higher GGM values, as indicated by negative values of $\mathcal{G}\left(\left|\psi_{F M S V}^{s u b\left\{m_{1}, m_{3}=n\right\}}\right\rangle\right)-$ $\mathcal{G}\left(\left|\psi_{F M S V}^{s u b\left\{m_{1}, m_{2}=n\right\}}\right\rangle\right)$ (see Fig. 6 (b) with $m_{1}$ and $n$ ).
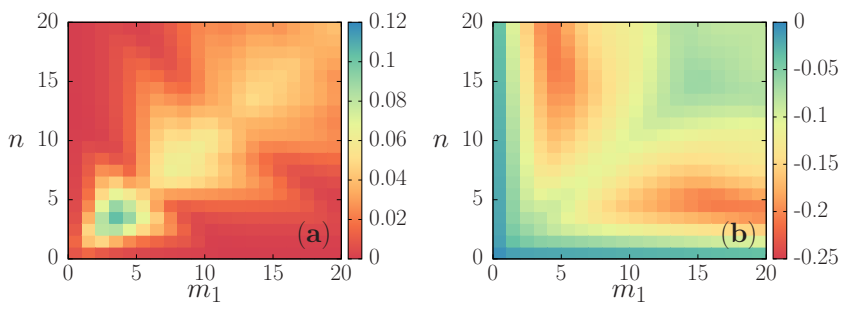

FIG. 6: (Color Online) Map of GGM in the $\left(m_{1}, n\right)$-plane. (a) $m_{1}$ and $m_{2(3)}=n$ photons are added in modes. To understand whether adding photons in adjacent modes can generate more multimode entanglement than that of the alternate mode, we plot $\left.\mathcal{G}\left(\left|\psi_{F M S V}^{a d d\left\{m_{1}, m_{3}=n\right\}}\right\rangle\right)\right)-\mathcal{G}\left(\left|\psi_{F M S V}^{a d d\left\{m_{1}, m_{2}=n\right\}}\right\rangle\right)$. We find that the adding photons in the alternate modes is beneficial than that of the adjacent modes. In (b), subtraction of photons is considered. Negative value of the quantity, $\left.\mathcal{G}\left(\left|\psi_{F M S V}^{s u b\left\{m_{1}, m_{3}=n\right\}}\right\rangle\right)\right)-\mathcal{G}\left(\left|\psi_{F M S V}^{s u b\left\{m_{1}, m_{2}=n\right\}}\right\rangle\right)$, indicates that the picture is opposite than the addition, thereby specifying that subtracting photons in alternate mode is an advantageous enterprise. Here $\mathrm{r}$ is fixed to 0.4 .

- For constrained photon operations, we fix the total number of photons added (subtracted) in either the adjacent or the alternate modes. Here we fix $m_{1}+m_{2(3)}=20$ and in Fig. $7(\mathrm{a})$, we consider the photon addition oper-
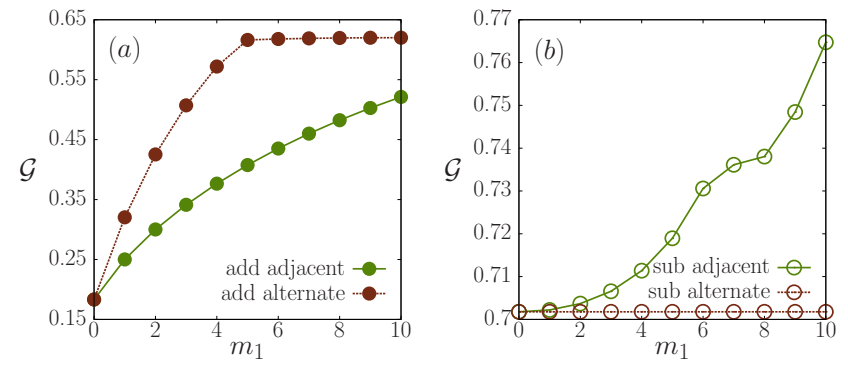

FIG. 7: ( Color online) GGM vs. $m_{1}$ with $m_{1}+m_{2}=20$. (a) GGM is measured after addition of 20 photons in the adjacent, $m_{1}$ and $m_{2}$ (solid circles) and in the alternate, $m_{1}$ and $m_{3}$, (solid squares) modes. (b) GGM is plotted after subtracting photons from the adjacent (hollow circles) and the alternate (hollow squares) modes. Here $r=0.4$. When photons are subtracted from the alternate modes, GGM remains constant with $m_{1}$, we call such a phenomena as freezing, as proven in Sec. IVC.

ation, and plot $\mathcal{G}\left(\left|\psi_{F M S V}^{a d d\left\{m_{1}, m_{2}=20-m_{1}\right\}}\right\rangle\right)$, indicated by the curve with solid green circles, and $\mathcal{G}\left(\left|\psi_{F M S V}^{a d d\left\{m_{1}, m_{3}=20-m_{1}\right\}}\right\rangle\right)$, represented by the curve with solid brown circles, with respect to $m_{1}$. In this situation, the alternate modes lead to higher elevation of GGM than that obtained for operations in the adjacent modes.

This trend is qualitatively different when we consider photon subtraction (as depicted in Fig. 7(b)). We find that $\mathcal{G}\left(\left|\psi_{F M S V}^{s u b\left\{m_{1}, m_{2}=20-m_{1}\right\}}\right\rangle\right)$ increases with increasing $m_{1}$ ( hollow green circles in Fig. $7(\mathrm{~b}))$, while $\mathcal{G}\left(\left|\psi_{F M S V}^{\operatorname{sub}\left\{m_{1}, m_{3}=20-m_{1}\right\}}\right\rangle\right)$ remains constant with the increase of $m_{1}$ (hollow red circles in Fig. 7(b)). We describe this feature as freezing of GGM and will provide an analytical analysis of the same in Sec. IV C. Note that in case of photonaddition, $\mathcal{G}$ slowly varies with high values of $m_{1}$ ( as depicted in $7(\mathrm{a})$ ) and so it is not similar to the freezing phenomena observed.

3. Multimode operations: Let us now consider a situation where three modes are involved in the photonaddition or -subtraction scheme. Let us fix $m_{1}+$ $m_{2}+m_{3}=20$, where $m_{1}, m_{2}$, and $m_{3}$ denote the numbers of photons added (subtracted) in the first, second and third mode respectively. As depicted in Fig. 8, the characteristics of GGM in this scenario is qualitatively different for addition and subtraction. Specifically, in case of photon-addition, we find that the GGM reaches its maximal value when all the three modes possess almost equal number of photons. However, in case of subtraction, maximal value of GGM is independent of $m_{1}$, i.e., we obtain freezing along $m_{1}$ which can also be explained analytically by the Theorem below. 

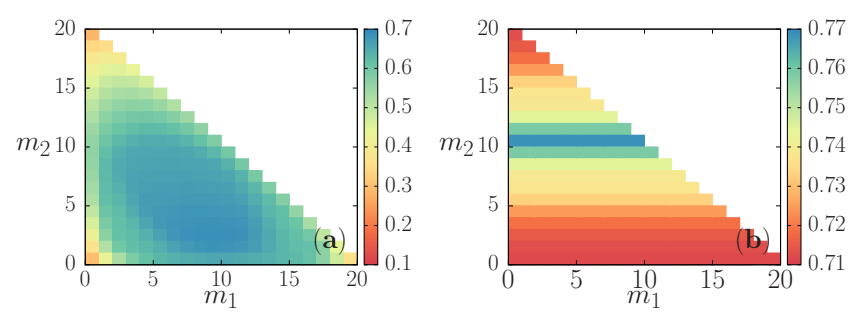

FIG. 8: GGM with respect to $m_{1}$ and $m_{2}$, when total number of photons added (subtracted) in three modes is fixed to 20 i.e., $m_{1}+m_{2}+m_{3}=20$. (a) corresponds to addition of photons. GGM is generated when equal number of photons are added in all the three modes. In (b), subtraction of photons is plotted with $m_{1}$ and $m_{2}$. For a fixed $m_{2}$, we find that the GGM remains constant with $m_{1}+m_{3}$, the freezing phenomena, similar to the one obtained in Fig. 7(b) (see also Theorem 2 in Sec. IVC). Here $r=0.4$.

\section{Freezing of GGM}

Let us show that the inherent form of the photonsubtracted state involving alternated modes ensures the freezing feature of GGM with the number of photons subtracted. Specifically, we obtain the following:

Theorem 2. In case of constant total number of photons subtracted from two alternate modes, the genuine multimode entanglement of a non-Gaussian FMSV state is independent of the number of photons subtracted from any one of those modes.

Proof. Without loss of generality, we assume that a fixed total number photons are subtracted from modes 1 and 3 , i.e., $m_{1}+m_{3}=$ constant, and no photons are added or subtracted from second and fourth modes. To prove our claim, we will now show that the coefficients of the photon-subtracted state, given in Eq. (38) is only a function of the total number of photons subtracted from the alternate modes, $m_{1}+m_{3}=M$, say. By putting $m_{2}=m_{4}=0$, Eq. (38) reduces to

$$
\begin{aligned}
\left|\psi_{F M S V}^{\text {sub }}\right\rangle= & \frac{1}{N^{\text {sub }}} \sum_{n=M}^{\infty} \sum_{r_{1}=m_{3}}^{n-m_{1}} \sum_{r_{2}=0}^{n}\left(\frac{1}{2} \tanh r\right)^{n} \sqrt{\left(\begin{array}{c}
n \\
r_{1}
\end{array}\right)} \sqrt{\left(\begin{array}{c}
n \\
r_{2}
\end{array}\right)} \sqrt{\frac{\left(n-r_{1}\right) !}{\left(n-r_{1}-m_{1}\right) !}} \sqrt{\frac{r_{1} !}{\left(r_{1}-m_{3}\right) !}} \\
& \left|n-r_{1}-m_{1}\right\rangle\left|n-r_{2}\right\rangle\left|r_{1}-m_{3}\right\rangle\left|r_{2}\right\rangle, \\
& \frac{1}{N^{\text {sub }}} \sum_{n=0}^{\infty} \sum_{r_{1}=0}^{n} \sum_{r_{2}=0}^{n}\left(\frac{1}{2} \tanh r\right)^{n+M} \sqrt{\left(\begin{array}{c}
n+M \\
r_{1}+m_{3}
\end{array}\right)} \sqrt{\left(\begin{array}{c}
n \\
r_{2}
\end{array}\right)} \sqrt{\frac{\left(n+m_{1}-r_{1}\right) !}{\left(n-r_{1}\right) !}} \sqrt{\frac{\left(r_{1}+m_{3}\right) !}{r_{1} !}} \\
= & \frac{1}{N^{\text {sub }}} \sum_{n=0}^{\infty} \sum_{r_{1}=0}^{n} \sum_{r_{2}=0}^{n}\left(\frac{1}{2} \tanh r\right)^{n+M} \sqrt{\left(\begin{array}{c}
n \\
r_{2}
\end{array}\right)} \sqrt{\frac{(n+M) !}{\left(n-r_{1}\right) ! r_{1} !}}\left|n-r_{1}\right\rangle\left|n-r_{2}\right\rangle\left|r_{1}\right\rangle\left|r_{2}\right\rangle .
\end{aligned}
$$

In the second equality, we make the change of variables, namely $r_{1} \rightarrow r_{1}+m_{3}$ and $n \rightarrow n+M$. From Eq. (41), it is clear that the above state is only function of $M$, and hence independent of $m_{1}$ and $m_{3}$ individually, but only dependent on their sum.

Remark: Note that the above theorem is proven for $m_{1}+m_{3}=M$ and $m_{2}=0$. Fig. 8 (b) shows that Theorem 2 also holds for the scenario when $m_{1}+m_{3}=M$ and $m_{2}=c$, where $c$ is a fixed integer.

\section{Analyzing GGM enhancement via non-Gaussianity}

In this section, we will try to figure out the departure of a photon added and subtracted states from the initial Gaussian FMSV state in terms of the relative entropy based measures of non-Gaussianity. Our main motive is to answer the following question:

Is there any connection between non-Gaussianity and GGM?

To address the above question, whether the GGM amplification obtained via addition and subtraction of photons has any connection with the non-Gaussianity, we first restate the definition of the measure. Specifically, the measure non-Gaussianity of a given state, $\rho$, in a CV system 


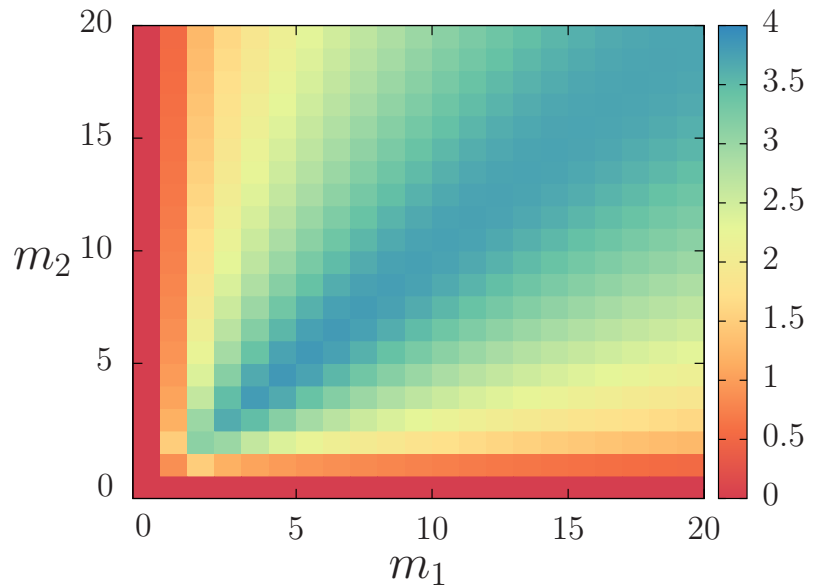

FIG. 9: Difference between non-Gaussianity, $\delta_{N G}\left(\left|\psi_{F M S V}^{a d d\left\{m_{1}, m_{2}\right\}}\right\rangle\right)-\delta_{N G}\left(\left|\psi_{F M S V}^{s u b\left\{m_{1}, m_{2}\right\}}\right\rangle\right)$, of the photonadded and -subtracted states against $\left(m_{1}, m_{2}\right)$-plane. We observe that for single mode operations, addition and subtraction of photons lead to the same amount of nonGaussianity, while for two mode operations, photon addition leads to greater non-Gaussianity. The squeezing parameter is fixed to 0.4 .

\begin{tabular}{|c|c|c|c|c|}
\hline$m_{1}, m_{2}$ & $\delta_{N G}^{\text {add }}$ & $f_{\mathcal{G}}^{\text {add }}$ & $\delta_{N G}^{\text {sub }}$ & $f_{\mathcal{G}}^{\text {sub }}$ \\
\hline \hline 2,0 & 2.7548 & 0.1639 & 2.7548 & 1.8234 \\
\hline 5,0 & 3.9001 & 0.3792 & 3.9001 & 4.0378 \\
\hline 10,0 & 4.8344 & 0.7293 & 4.8344 & 6.719 \\
\hline 2,1 & 4.5661 & 0.7584 & 2.0653 & 2.9408 \\
\hline 5,1 & 5.5072 & 1.0780 & 3.6208 & 4.7684 \\
\hline 10,1 & 6.1742 & 1.5493 & 4.6930 & 7.1720 \\
\hline
\end{tabular}

TABLE I: Comparative study of fractional enhancement of GGM from FMSV value and non-Gaussianity on addition or subtraction of photons from a single and two modes of the FMSV state. Note that $\delta_{N G}^{a d d / s u b} \equiv \delta_{N G}\left(\left|\psi_{F M S V}^{a d d / s u b\left\{m_{1}, m_{2}\right\}}\right\rangle\right)$. The squeezing parameter is fixed to 0.4 .

is defined in terms of relative entropy distance, as [80-82]

$$
\delta_{N G}(\rho)=\min _{\tau_{G}} S\left(\rho \| \tau_{G}\right),
$$

where the minimization is taken over all possible Gaussian states $\tau_{G}$, lying in the same Hilbert space of $\rho$. The Gaussian state, which achieve the minimum possess the same displacement vector and covariance matrix as $\rho$, lets consider it as $\rho_{G}$, hence

$$
\delta_{N G}(\rho)=S\left(\rho \| \rho_{G}\right)=S(\rho)-S\left(\rho_{G}\right),
$$

where $S(\sigma)=-\operatorname{tr} \sigma \log _{2} \sigma$. We find that $\delta_{N G}$ increases both for photon-added and -subtracted states. Therefore, the enhancement of GGM seems to be consistent with the increase of non-Gaussianity as the number of photons added (subtracted) increases, and thus can be plausibly argued as the physical reason behind the observed improvements. However, a more detailed analysis reveals that in most cases (see Fig. 9), photon addition leads to much faster de-Gaussification compared to photon subtraction although the GGM content of the photonsubtracted state is higher than that of the photon-added ones, see Figs. 4 and 5. So, non-Gaussianity cannot explain the difference in response of photon addition and subtraction. We would like to mention here that the physical reason for the different behaviour obtained for photon addition and subtraction remained unanswered in the earlier works as well [65, 66, 76-79].

Nevertheless, a quantitative analysis is carried out between non-Gaussianity and fractional enhancement of GGM for the photons-added (-subtracted) states where the later one is defined as

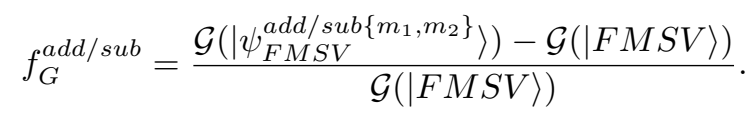

It allows us to track how fast the GGM grows on addition or subtraction of photons, see Table. I.

To summarize, Fig. 9 and Table. I reveal three distinct features -(1) when photons are added (subtracted) in a single mode, one gets the same amount of nonGaussianity; (2) for two mode operations, photon addition always leads to higher non-Gaussianity compared to the photon subtraction; (3) in both the cases, GGM content in photon-subtracted states from FMSV is higher than that of the photon-added states, thereby showing $f_{G}^{s u b}>f_{G}^{a d d}$. Therefore, non-Gaussianity cannot conclusively discriminate photon addition and subtraction according to their genuine multomide entanglement. Notice also that the difference between entanglement values of photon-added and -subtracted states cannot be explained by other physical quantities like departure of the photon- added (-subtracted) state from the initial Gaussian FMSV state, the fidelity of photon-added (subtracted) state with a FMSV state having the same value of GGM [83]. We believe that a complete satisfactory and ubiquitous physical reasoning may depend on the structure of the state itself, on which mode(s) the photonic operations are performed, and on the quantity of interest.

\section{CONCLUSION}

The generalized geometric measure (GGM), which owes its origin to the geometry of quantum state space, has established itself as a computationally efficient quantifier of genuine multiparty entanglement of pure states in finite dimensional systems. Based on Schmidt decomposition, its applicability can also be extended to compute genuine multimode entanglement between the various modes of a multimode pure state consisting of infinite dimensional subsystems. 
Nevertheless, such simplified formula can also be intractable due to the infinite dimensional structures inherent in these systems. However, we proved that for Gaussian multimode pure states, GGM can be expressed in terms of the symplectic eigenvalues of the covariance matrices obtained from the various relevant reduced states. Essentially, we are able to compute the coverted maximal eigenvalue in terms of the symplectic invariants and hence this simplification. To illustrate the functionality of the formula, we computed GGM of some prototypical three- and four-mode Gaussian states. Note that, the formula for GGM is efficiently scalable, can be employed to evaluate GGM of Gaussian states with higher number of modes, and its pertinence is not merely restricted to three- and four-mode states. Furthermore, the symplectic eigenvalues are evaluated from the covariance matrices which are in turn composed of quadrature correlations, the typical quantities extracted in experiments. This experimental friendliness adds another point of merit to our work.

When one proceeds beyond the Gaussian paradigm, such simplified evaluation of GGM is not possible. However, for some non-Gaussian states (like photon-added and -subtracted states), symmetries in the structure of the states enables to find maximal eigenvalue of all reduced states. De-Gaussification via photon addition and subtraction displays substantial enhancement of GGM from the Gaussian value. We performed a comparative study in the increase of genuine multimode entanglement induced by addition and subtraction in the three- and four-mode scenarios and observe some novel features. A careful analysis also revealed that although the increase of genuine multimode entanglement content of photonadded (-subtracted) states over the four mode squeezed vacuum states can be answered by the increase of the distance-based non-Gaussianity measure, the superiority in terms of multimode entanglement value obtained for photon subtraction compared to photon-addition cannot be explained by the non-Gaussianity measure.

To summarize, our work sheds light on the quantification of genuine multimode entanglement in continuous variable systems. We believe, our work will be a stepping stone for further systematic analysis of multimode entanglement involving non-Gaussian states and mixed states.

\section{Acknowledgments}

TD thanks Prof. Marek Zukowski and Bianka Woloncewicz for insightful comments and discussion and acknowledge The International Centre for Theory of Quantum Technologies project (contract no. 2018/MAB/5).

\section{Appendix A: Schmidt decomposition in CV system}

In this section, we are trying to investigate that if in the continuous variable system, matrix diagonalization is possible, then the Schmidt decomposition is also possible.

Statement: Suppose $A$ is a $d$ dimensional normal matrix i.e., $A A^{\dagger}=A^{\dagger} A$. The Hermitian matrix $H^{\dagger}=H$ and unitary matrix $U^{\dagger} U=I$ are the example of normal matrices. There always exists, an unitary matrix $U$, such that $U A U^{\dagger}=A_{\text {diag }}$, where $A_{\text {diag }}$ is a diagonal matrix, i.e, $A_{i j}=\delta_{i j} a_{i}$. The above statement is also true in infinite dimensions provided the operator is compact and Hermitian.

\section{Proof of Schmidt decomposition in infinite dimension}

Suppose $\left|\psi_{12}\right\rangle$, is a two-mode continuous variable system. $\left|\psi_{12}\right\rangle \in \mathcal{H}^{1} \otimes \mathcal{H}^{2}, \mathcal{H}^{1}\left(\mathcal{H}^{2}\right)$ are two infinite dimensional Hilbert spaces, with countably infinite number of fock basis $\{|n\rangle\}$ and $\{|m\rangle\}$. Hence, it can be expanded as

$$
\left|\psi_{12}\right\rangle=\sum_{m, n=0}^{\infty} a_{m, n}|m, n\rangle
$$

where $a_{m, n}$ is the complex coefficient, satisfy $\sum_{m, n=0}^{\infty}\left|a_{m, n}\right|^{2}=1$. Let us consider a single-mode density matrix as

$$
\begin{aligned}
\rho_{1}=\operatorname{tr}_{2}\left(\left|\psi_{12}\right\rangle\left\langle\psi_{12}\right|\right) & =\sum_{m, m^{\prime}}^{\infty}\left(\sum_{n} a_{m, n} a_{m^{\prime}, n}^{*}\right)|m\rangle\left\langle m^{\prime}\right| \\
& =\sum_{m, m^{\prime}}^{\infty} C_{m, m^{\prime}}|m\rangle\left\langle m^{\prime}\right|
\end{aligned}
$$

where $C_{m, m^{\prime}}=\sum_{n}^{\infty} a_{m, n} a_{m^{\prime}, n}^{*}$. It is clear that $C^{\dagger}=C$, i.e., $C$ is a Hermitian matrix. Moreover $C$ is also a compact matrix which guarantees that the spectral theorem can also be applied here [72]. Hence we have an unitary $U$, such that $U C U^{\dagger}=\Lambda_{\text {diag }}$. Hence,

$$
\begin{aligned}
\rho_{1}=\sum_{m, m^{\prime}} C_{m, m^{\prime}}|m\rangle\left\langle m^{\prime}\right| & =\sum_{m, m^{\prime}, \mu} U_{m, \mu} \lambda_{\mu} U_{m^{\prime}, \mu}^{*} \lambda_{\mu}|m\rangle\left\langle m^{\prime}\right| \\
& =\sum_{\mu} \lambda_{\mu}|\mu\rangle\langle\mu|
\end{aligned}
$$

where $|\mu\rangle=\sum_{m} U_{m, \mu}|m\rangle . \quad \lambda_{\mu}$ s are the eigenvalue of $\rho_{1}$ and $\{|\mu\rangle\}$ is the eigenbasis or the Schmidt basis.

Now we can always write the initial pure state as $\left|\psi_{12}\right\rangle=\sum_{\mu}|\mu\rangle_{1}|\tilde{\mu}\rangle_{2}$, where $|\tilde{\mu}\rangle={ }_{1}\left\langle\mu \mid \psi_{12}\right\rangle$, clearly $|\tilde{\mu}\rangle$ is not a normalized state. To prove that this expression is the schmidt decomposition of $\left|\psi_{12}\right\rangle$, we need to prove $\{|\bar{\mu}\rangle\}$ is mutually orthogonal. 


$$
\begin{aligned}
|\tilde{\mu}\rangle={ }_{1}\left\langle\mu \mid \psi_{12}\right\rangle & =\left(\sum_{m^{\prime}} U_{m^{\prime}, \mu}^{*}\left\langle m^{\prime}\right|\right)\left(\sum_{m, n=0}^{\infty} a_{m, n}|m, n\rangle\right) \\
& =\sum_{m, n} U_{m, \mu}^{*} a_{m n}|n\rangle_{2}
\end{aligned}
$$

Now

$$
\begin{aligned}
\langle\tilde{\nu} \mid \tilde{\mu}\rangle & =\sum_{m^{\prime}, n^{\prime}} U_{m^{\prime}, \nu} a_{m^{\prime} n^{\prime 2}}^{*}\left\langle n^{\prime}\right|\left(\sum_{m, n} U_{m, \mu}^{*} a_{m n}|n\rangle_{2}\right) \\
& =\sum_{m^{\prime}, m} U_{m^{\prime}, \nu} \sum_{n} a_{m^{\prime} n}^{*} a_{m n} U_{m, \mu}^{*} \\
& =\sum_{m^{\prime}, m} U_{m^{\prime}, \nu} C_{m, m^{\prime}} U_{m, \mu}^{*}=\left(U^{\dagger} C U\right)_{\nu, \mu}=\delta_{\mu, \nu} \lambda_{\mu},
\end{aligned}
$$

By choosing $|\tilde{\mu}\rangle=\sqrt{\lambda_{\mu}}|\mu\rangle$, we get the Schmidt decomposition of a pure state $\left|\psi_{12}\right\rangle$ in CV system as

$$
\left|\psi_{12}\right\rangle=\sum_{\mu}^{\infty} \lambda_{\mu}|\mu, \mu\rangle
$$

\section{Appendix B: Covariance matrix of the FMSV state}

The FMSV state [66] possess the following covariance matrix:

$$
\begin{gathered}
\Lambda^{\mathrm{FMSV}}= \\
\frac{1}{2}\left[\begin{array}{cccc}
\cosh ^{2} r \mathbb{I}_{2} & \frac{1}{2} \sinh 2 r \sigma_{z} & \sinh ^{2} r \mathbb{I}_{2} & \frac{1}{2} \sinh 2 r \sigma_{z} \\
\frac{1}{2} \sinh _{2} r \sigma_{z} & \cosh ^{2} r \mathbb{I}_{2} & \frac{1}{2} \sinh 2 r \sigma_{z} & \sinh ^{2} r \mathbb{I}_{2} \\
\sinh ^{2} r \mathbb{I}_{2} & \frac{1}{2} \sinh _{2} r \sigma_{z} & \cosh ^{2} r \mathbb{I}_{2} & \frac{1}{2} \sinh 2 r \sigma_{z} \\
\frac{1}{2} \sinh 2 r \sigma_{z} & \sinh ^{2} r \mathbb{I}_{2} & \frac{1}{2} \sinh 2 r \sigma_{z} & \cosh ^{2} r \mathbb{I}_{2}
\end{array}\right],
\end{gathered}
$$

where $\mathbb{I}_{2}=\operatorname{diag}\{1,1\}$, and $\sigma_{z}=\operatorname{diag}\{1,-1\}$. All the single-mode reduced covariance matrices are identical and is given by, $\Lambda_{\text {single }}^{\mathrm{FMSV}}=\frac{1}{2} \cosh ^{2} r \mathbb{I}_{2}$. The relevant two-mode reduced covariance matrices, corresponding to the adjacent and alternate modes are given by

$$
\Lambda_{\text {adjacent }}^{\mathrm{FMSV}}=\frac{1}{2}\left[\begin{array}{cc}
\cosh ^{2} r \mathbb{I}_{2} & \frac{1}{2} \sinh 2 r \sigma_{z} \\
\frac{1}{2} \sinh 2 r \sigma_{z} & \cosh ^{2} r \mathbb{I}_{2}
\end{array}\right],
$$

and

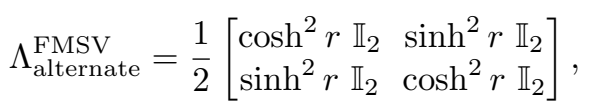

respectively.
[1] R. Horodecki, P. Horodecki, M. Horodecki, and K. Horodecki, Rev. Mod. Phys. 81, 865 (2009), URL https://link.aps.org/doi/10.1103/RevModPhys . 81. 865.

[2] E. Schrdinger, Mathematical Proceedings of the Cambridge Philosophical Society 31, 555563 (1935).

[3] C. H. Bennett, G. Brassard, C. Crépeau, R. Jozsa, A. Peres, and W. K. Wootters, Phys. Rev. Lett. 70, 1895 (1993), URL https://link.aps.org/doi/10. 1103/PhysRevLett. 70.1895.

[4] D. Bouwmeester, J.-W. Pan, K. Mattle, M. Eibl, H. Weinfurter, and A. Zeilinger, Nature 390, 575 (1997), URL https://doi.org/10.1038/37539.

[5] S. Pirandola, J. Eisert, C. Weedbrook, A. Furusawa, and S. L. Braunstein, Nature Photonics 9, 641 (2015), URL https://doi.org/10.1038/nphoton.2015.154.

[6] C. H. Bennett and S. J. Wiesner, Phys. Rev. Lett. 69, 2881 (1992), URL https://link.aps.org/doi/10. 1103/PhysRevLett.69.2881.

[7] Y. Guo, B.-H. Liu, C.-F. Li, and G.-C. Guo, Advanced Quantum Technologies 2, 1900011 (2019), URL https: //doi.org/10.1002/qute.201900011.

[8] A. K. Ekert, Phys. Rev. Lett. 67, 661 (1991), URL https://link.aps.org/doi/10.1103/PhysRevLett.67. 661.

[9] C. H. Bennett, G. Brassard, and N. D. Mermin, Phys.
Rev. Lett. 68, 557 (1992), URL https ://link.aps.org/ doi/10.1103/PhysRevLett.68.557.

[10] L.-A. Wu, M. S. Sarandy, and D. A. Lidar, Phys. Rev. Lett. 93, 250404 (2004), URL https://link.aps.org/ doi/10.1103/PhysRevLett.93.250404.

[11] F. Verstraete, M. A. Martín-Delgado, and J. I. Cirac, Phys. Rev. Lett. 92, 087201 (2004), URL https://Iink. aps.org/doi/10.1103/PhysRevLett.92.087201.

[12] B. K. Chakrabarti, A. Dutta, and P. Sen, Quantum Ising phases and transitions in transverse Ising models (Springer Berlin Heidelberg, 1996), ISBN 9783540498650, URL https://doi.org/10.1007/ 978-3-540-49865-0.

[13] S. Sachdev, Quantum Phase Transitions (Cambridge University Press, 2009), URL https://doi.org/10. $1017 /$ cbo9780511973765.

[14] A. SHIMONY, Annals of the New York Academy of Sciences 755, 675 (1995), URL https://doi.org/10.1111/ j.1749-6632.1995.tb39008.x.

[15] D. C. Brody and L. P. Hughston, Journal of Geometry and Physics 38, 19 (2001), URL https://doi.org/10. 1016/s0393-0440(00)00052-8.

[16] H. Barnum and N. Linden, Journal of Physics A: Mathematical and General 34, 6787 (2001), URL https: //doi.org/10.1088/0305-4470/34/35/305.

[17] D. A. Meyer and N. R. Wallach, Journal of Mathemati- 
cal Physics 43, 4273 (2002), URL https://doi.org/10. 1063/1.1497700.

[18] T.-C. Wei and P. M. Goldbart, Phys. Rev. A 68, 042307 (2003), URL https://link.aps.org/doi/10. 1103/PhysRevA.68.042307.

[19] A. Osterloh and J. Siewert, Phys. Rev. A 72, 012337 (2005), URL https://link.aps.org/doi/10. 1103/PhysRevA.72.012337.

[20] R. Orús, Phys. Rev. Lett. 100, 130502 (2008), URL https://link.aps.org/doi/10.1103/PhysRevLett. 100.130502 .

[21] R. Orús, Phys. Rev. A 78, 062332 (2008), URL https: //link.aps.org/doi/10.1103/PhysRevA.78.062332.

[22] D. Ž. Doković and A. Osterloh, Journal of Mathematical Physics 50, 033509 (2009), URL https://doi.org/10. 1063/1.3075830.

[23] Q.-Q. Shi, R. Orús, J. O. Fjærestad, and H.-Q. Zhou, New Journal of Physics 12, 025008 (2010), URL https: //doi.org/10.1088/1367-2630/12/2/025008.

[24] R. Orús and T.-C. Wei, Phys. Rev. B 82, 155120 (2010), URL https://link.aps.org/doi/10.1103/PhysRevB. 82. 155120.

[25] V. Coffman, J. Kundu, and W. K. Wootters, Phys. Rev. A 61, 052306 (2000), URL https://link.aps.org/doi/ 10.1103/PhysRevA.61.052306.

[26] T. J. Osborne and F. Verstraete, Phys. Rev. Lett. 96, 220503 (2006), URL https://link.aps.org/doi/ 10.1103/PhysRevLett.96.220503.

[27] G. Adesso, A. Serafini, and F. Illuminati, Phys. Rev. A 73, 032345 (2006), URL https://link.aps.org/doi/ 10.1103/PhysRevA.73.032345.

[28] T. Hiroshima, G. Adesso, and F. Illuminati, Phys. Rev. Lett. 98, 050503 (2007), URL https://Iink.aps.org/ doi/10.1103/PhysRevLett.98.050503.

[29] Y.-C. Ou and H. Fan, Phys. Rev. A 75, 062308 (2007), URL https://link.aps.org/doi/10.1103/PhysRevA. 75.062308.

[30] A. Kay, D. Kaszlikowski, and R. Ramanathan, Phys. Rev. Lett. 103, 050501 (2009), URL https://link. aps . org/doi/10.1103/PhysRevLett.103.050501.

[31] M. Hayashi and L. Chen, Phys. Rev. A 84, 012325 (2011), URL https://link.aps.org/doi/10. 1103/PhysRevA.84.012325.

[32] F. F. Fanchini, M. F. Cornelio, M. C. de Oliveira, and A. O. Caldeira, Phys. Rev. A 84, 012313 (2011), URL https://link.aps.org/doi/10.1103/PhysRevA. 84.012313.

[33] A. Streltsov, G. Adesso, M. Piani, and D. Bruß, Phys. Rev. Lett. 109, 050503 (2012), URL https://link.aps. org/doi/10.1103/PhysRevLett.109.050503.

[34] A. Kumar, R. Prabhu, A. Sen(De), and U. Sen, Phys. Rev. A 91, 012341 (2015), URL https: //link. aps.org/ doi/10.1103/PhysRevA.91.012341.

[35] S. Roy, T. Das, A. Kumar, A. Sen(De), and U. Sen, Phys. Rev. A 98, 012310 (2018), URL https: //link. aps.org/ doi/10.1103/PhysRevA.98.012310.

[36] S. Rethinasamy, S. Roy, T. Chanda, A. Sen(De), and U. Sen, Phys. Rev. A 99, 042302 (2019), URL https: //link.aps.org/doi/10.1103/PhysRevA.99.042302.

[37] A. Sen(De) and U. Sen, Phys. Rev. A 81, 012308 (2010), URL https://link.aps.org/doi/10.1103/PhysRevA. 81.012308.

[38] A. Shimony, Annals of the New York Academy of Sciences 755, 675 (1995), URL https://doi.org/10.1111/ j.1749-6632.1995.tb39008.x.

[39] H. Barnum and N. Linden, J. Phys. A: Mathematical and General 34, 6787 (2001), URL http://stacks.iop.org/ $0305-4470 / 34 / i=35 / a=305$.

[40] T.-C. Wei and P. M. Goldbart, Phys. Rev. A 68, 042307 (2003), URL https://link.aps.org/doi/10. 1103/PhysRevA.68.042307.

[41] M. Blasone, F. Dell'Anno, S. De Siena, and F. Illuminati, Phys. Rev. A 77, 062304 (2008), URL https://link. aps.org/doi/10.1103/PhysRevA.77.062304.

[42] R. Prabhu, S. Pradhan, A. Sen(De), and U. Sen, Phys. Rev. A 84, 042334 (2011), URL https://Iink.aps.org/ doi/10.1103/PhysRevA.84.042334.

[43] H. S. Dhar, A. Sen(De), and U. Sen, Phys. Rev. Lett. 111, 070501 (2013), URL https://link.aps.org/doi/ 10.1103/PhysRevLett.111.070501.

[44] L. Jindal, A. D. Rane, H. S. Dhar, A. Sen(De), and U. Sen, Phys. Rev. A 89, 012316 (2014), URL https: //link.aps.org/doi/10.1103/PhysRevA.89.012316.

[45] A. Biswas, R. Prabhu, A. Sen(De), and U. Sen, Phys. Rev. A 90, 032301 (2014), URL https://link.aps.org/ doi/10.1103/PhysRevA.90.032301.

[46] T. Das, R. Prabhu, A. Sen(De), and U. Sen, Phys. Rev. A 90, 022319 (2014), URL https://link.aps.org/doi/ 10.1103/PhysRevA.90.022319.

[47] T. Das, S. S. Roy, S. Bagchi, A. Misra, A. Sen(De), and U. Sen, Phys. Rev. A 94, 022336 (2016), URL https: //link.aps.org/doi/10.1103/PhysRevA.94.022336.

[48] S. L. Braunstein and P. van Loock, Rev. Mod. Phys. 77, 513 (2005), URL https://link.aps.org/doi/10.1103/ RevModPhys.77.513.

[49] S. L. Braunstein and A. K. Pati, eds., Quantum Information with Continuous Variables (Springer Netherlands, 2003), URL https://doi.org/10.1007/ 978-94-015-1258-9.

[50] G. Adesso, S. Ragy, and A. R. Lee, Open Systems \& Information Dynamics 21, 1440001 (2014), URL https: //doi.org/10.1142/s1230161214400010.

[51] C. Weedbrook, S. Pirandola, R. García-Patrón, N. J. Cerf, T. C. Ralph, J. H. Shapiro, and S. Lloyd, Rev. Mod. Phys. 84, 621 (2012), URL https://link.aps.org/doi/ 10.1103/RevModPhys.84.621.

[52] X. WANG, T. HIROSHIMA, A. TOMITA, and M. HAYASHI, Physics Reports 448, 1 (2007), URL https://doi.org/10.1016/j.physrep.2007.04.005.

[53] I. L. Chuang, D. W. Leung, and Y. Yamamoto, Phys. Rev. A 56, 1114 (1997), URL https://link.aps.org/ doi/10.1103/PhysRevA.56.1114.

[54] S. Lloyd and S. L. Braunstein, Phys. Rev. Lett. 82, 1784 (1999), URL https://link.aps.org/doi/10. 1103/PhysRevLett.82.1784.

[55] S. D. Huver, C. F. Wildfeuer, and J. P. Dowling, Phys. Rev. A 78, 063828 (2008), URL https://link.aps.org/ doi/10.1103/PhysRevA.78.063828.

[56] J. Eisert, S. Scheel, and M. B. Plenio, Phys. Rev. Lett. 89, 137903 (2002), URL https://link.aps.org/doi/ 10.1103/PhysRevLett.89.137903.

[57] G. Giedke and J. Ignacio Cirac, Phys. Rev. A 66, 032316 (2002), URL https://link.aps.org/doi/10. 1103/PhysRevA.66.032316.

[58] K. K. Sabapathy, J. S. Ivan, and R. Simon, Phys. Rev. Lett. 107, 130501 (2011), URL https://link.aps.org/ doi/10.1103/PhysRevLett.107.130501.

[59] J. Niset, J. Fiurášek, and N. J. Cerf, Phys. Rev. Lett. 
102, 120501 (2009), URL https://link.aps.org/doi/ 10.1103/PhysRevLett. 102.120501.

[60] G. Adesso, F. Dell'Anno, S. De Siena, F. Illuminati, and L. A. M. Souza, Phys. Rev. A 79, 040305 (2009), URL https://link.aps.org/doi/10. 1103/PhysRevA.79.040305.

[61] S. Zhang and P. van Loock, Phys. Rev. A 84, 062309 (2011), URL https://link.aps.org/doi/10. 1103/PhysRevA.84.062309.

[62] N. J. Cerf, O. Krüger, P. Navez, R. F. Werner, and M. M. Wolf, Phys. Rev. Lett. 95, 070501 (2005), URL https : // link.aps.org/doi/10.1103/PhysRevLett.95.070501.

[63] E. Wigner, Phys. Rev. 40, 749 (1932), URL https:// link.aps.org/doi/10.1103/PhysRev.40.749.

[64] Usha Devi, A. R., Prabhu, R., and Uma, M. S., Eur. Phys. J. D 40, 133 (2006), URL https://doi.org/10. 1140/epjd/e2006-00135-x.

[65] C. Navarrete-Benlloch, R. García-Patrón, J. H. Shapiro, and N. J. Cerf, Phys. Rev. A 86, 012328 (2012), URL https://link.aps.org/doi/10.1103/PhysRevA. 86.012328.

[66] T. Das, R. Prabhu, A. Sen(De), and U. Sen, Phys. Rev. A 93, 052313 (2016), URL https://link.aps.org/doi/ 10.1103/PhysRevA.93.052313.

[67] M. Walschaers, Y.-S. Ra, and N. Treps, Phys. Rev. A 100, 023828 (2019), URL https://link.aps.org/doi/ 10.1103/PhysRevA.100.023828.

[68] Y.-S. Ra, A. Dufour, M. Walschaers, C. Jacquard, T. Michel, C. Fabre, and N. Treps, Nature Physics 16, 144 (2019), URL https://doi.org/10.1038/ s41567-019-0726-y.

[69] V. I. Arnold, Mathematical Methods of Classical Mechanics (Springer New York, 1989), URL https://doi.org/ 10.1007/978-1-4757-2063-1.

[70] S. Kobayashi, Foundations of differential geometry (Wiley, New York, 1996), ISBN 978-0-471-15732-8.

[71] A. Botero and B. Reznik, Phys. Rev. A 67,
052311 (2003), URL https://link.aps.org/doi/10. 1103/PhysRevA.67.052311.

[72] F. Herbut, Quanta 7, 19 (2018), URL https://doi.org/ 10.12743/quanta.v7i1.69.

[73] Gaussian States in Quantum Information (Bibliopolis, 2005), ISBN 887088483X, URL https://www. xarg.org/ ref/a/887088483X/.

[74] L. E. Buchholz, T. Moroder, and O. Gühne, Annalen der Physik 528, 278 (2015), URL https://doi.org/10. 1002/andp. 201500293.

[75] M. Bondani, A. Allevi, E. Puddu, A. Andreoni, A. Ferraro, and M. G. A. Paris, Optics Letters 29, 180 (2004), URL https://doi.org/10.1364/o1.29.000180.

[76] Y. Yang, Journal of the Optical Society of America B 33, 2545 (2016), URL https://doi.org/10.1364/ josab. 33.002545.

[77] S. Roy, T. Chanda, T. Das, A. Sen(De), and U. Sen, Phys. Rev. A 98, 052131 (2018), URL https://link. aps.org/doi/10.1103/PhysRevA.98.052131.

[78] B. Mojaveri, A. Dehghani, and R. J. Bahrbeig, The European Physical Journal Plus 134 (2019), URL https: //doi.org/10.1140/epjp/i2019-12823-7.

[79] T. M. Duc and T. Q. Dat, Optik p. 164479 (2020), URL https://doi.org/10.1016/j.ijleo.2020.164479.

[80] M. G. Genoni, M. G. A. Paris, and K. Banaszek, Phys. Rev. A 78, 060303 (2008), URL https://link.aps.org/ doi/10.1103/PhysRevA.78.060303.

[81] M. G. Genoni and M. G. A. Paris, Phys. Rev. A 82, 052341 (2010), URL https://link.aps.org/doi/ 10.1103/PhysRevA.82.052341.

[82] P. Marian and T. A. Marian, Phys. Rev. A 88, 012322 (2013), URL https://link.aps.org/doi/10. 1103/PhysRevA.88.012322.

[83] T. J. Bartley and I. A. Walmsley, New Journal of Physics 17, 023038 (2015), URL https://doi.org/10. 1088\%2F $1367-2630 \% 2 \mathrm{~F} 17 \% 2 \mathrm{~F} 2 \% 2 \mathrm{~F} 023038$. 